\title{
Nicotine-Induced Structural Plasticity in Mesencephalic Dopaminergic Neurons Is Mediated by Dopamine D3 Receptors and Akt-mTORC1 Signaling
}

\author{
Ginetta Collo, Federica Bono, Laura Cavalleri, Laura Plebani, Stefania Mitola, Emilio Merlo \\ Pich, Mark J. Millan, Michele Zoli, Uwe Maskos, PierFranco Spano, and Cristina Missale \\ Department of Molecular and Translational Medicine (G.C., F.B., L.C., L.P., S.M., PF.S., C.M.) and Woman Health Center- \\ Camillo Golgi Foundation (G.C., F.B., L.C., PF.S., C.M.), University of Brescia, Brescia, Italy; Neuroscience Pharmaceutical \\ Division, Hoffman-La Roche, Basel, Switzerland (E.M.P.); Division of Psychopharmacology, Institut de Recherches Servier, \\ Croissy-Sur-Seine, France (M.J.M.); Department of Biomedical, Metabolic and Neural Sciences, University of Modena and \\ Reggio Emilia, Italy (M.Z.); and Unité de Neurobiologie Intégrative des Systèmes Cholinergiques, Centre National de la \\ Recherche Scientifique, Unité de Recherche Associée 2182, Institut Pasteur, Paris, France (U.M.)
}

Received January 22, 2013; accepted March 19, 2013

\begin{abstract}
Although long-term exposure to nicotine is highly addictive, one beneficial consequence of chronic tobacco use is a reduced risk for Parkinson's disease. Of interest, these effects both reflect structural and functional plasticity of brain circuits controlling reward and motor behavior and, specifically, recruitment of nicotinic acetylcholine receptors (nAChR) in mesencephalic dopaminergic neurons. Because the underlying cellular mechanisms are poorly understood, we addressed this issue with use of primary cultures of mouse mesencephalic dopaminergic neurons. Exposure to nicotine (1-10 $\mu \mathrm{M})$ for 72 hours in vitro increased dendritic arborization and soma size in primary cultures. These effects were blocked by mecamylamine and dihydro- $\beta$-erythroidine, but not methyllycaconitine. The involvement of $\alpha 4 \beta 2 \mathrm{nAChR}$ was supported by the lack of nicotineinduced structural remodeling in neurons from $\alpha 4$ null mutant mice (KO). Challenge with nicotine triggered phosphorylation of the extracellular signal-regulated kinase (ERK) and the thymoma viral proto-oncogene (Akt), followed by activation of the mammalian
\end{abstract}

target of rapamycin complex 1 (mTORC1)-dependent p70 ribosomal S6 protein kinase. Upstream pathway blockade using the phosphatidylinositol 3-kinase inhibitor LY294002 [2-(4-morpholinyl)8-phenyl-4H-1-benzopyran-4-one hydrochloride] resulted in suppression of nicotine-induced phosphorylations and structural plasticity. These effects were dependent on functional DA D3 receptor (D3R), because nicotine was inactive both in cultures from D3R KO mice and after pharmacologic blockade with D3R antagonist trans-N-4-2-(6-cyano-1,2,3, 4-tetrahydroisoquinolin2-yl)ethylcyclohexyl-4-quinolinecarboxamide (SB-277011-A) $(50 \mathrm{nM})$. Finally, exposure to nicotine in utero $(5 \mathrm{mg} / \mathrm{kg} /$ day for 5 days) resulted in increased soma area of DAergic neurons of newborn mice, effects not observed in D3 receptor null mutant mice mice. These findings indicate that nicotine-induced structural plasticity at mesencephalic dopaminergic neurons involves $\alpha 4 \beta 2$ nAChRs together with dopamine D3R-mediated recruitment of ERK/Akt-mTORC1 signaling.
This work was supported by the Italian Institute of Technology (Grant to C.M.); and Italian Project of Main National Interest [Grant 2009R7WCZS 006. (to G.C.)]. E.M.P. is a full time employee of Hoffman-La Roche; M.J.M. is a full time employee of Institut de Recherches Servier. The other authors declare no conflicts of interest in this work.

This work was previously presented in abstract form at the following conference: Collo G, Plebani L, Cavalleri L, Bono F, Merlo Pich E, Zoli M, Maskos U, Spano PF, Missale C (2011) Neuroadaptation mechanisms to nicotine in mesencephalic dopaminergic neurons: role of dopamine D3 receptor and nicotinic acetylcholine receptors. Neuroscience 2011; 2011 Nov 12-16; Washington, DC. Society for Neuroscience, Washington, D.C.

dx.doi.org/10.1124/mol.113.084863.

\section{Introduction}

Tobacco smoking is a pervasive habit that has been associated with pathologic addiction (Benowitz 1988) and reduced risk for Parkinson's disease (PD) (Grandinetti et al., 1994; Wirdefeldt et al., 2011). Of interest, in both conditions, a critical substrate was recognized to be the DAergic system, involving neurons located in the ventral tegmental area (VTA) (Corrigall et al., 1994) and the substantia nigra (SN) (Quik et al., 2006), respectively. Although an impressive amount of neuropharmacological

ABBREVIATIONS: 7-OH-DPAT, 7-hydroxy- $N, N$-di-propyl-2-aminotetralin; Akt, thymoma viral proto-oncogene; BDNF, brain-derived neurotrophic factor; BSA, bovine serum albumin; D3KO, D3 receptor null mutant mice; $\mathrm{DH} \beta \mathrm{E}$, dihydro- $\beta$-erythroidine; $\mathrm{E}$, embryonic day; $\mathrm{ERK}$, extracellular signal-regulated kinase; IR, immunoreactivity; KO, knockout; LY294002, 2-(4-morpholinyl)-8-phenyl-4H-1-benzopyran-4-one hydrochloride; MEC, mecamylamine; MEK, mitogen-activated protein kinase kinase; MLA, methyllycaconitine; mTOR, mammalian target of rapamycin; mTORC1, mammalian target of rapamycin complex 1; nAChR, nicotinic acetylcholine receptors; p70S6K, p70 ribosomal S6 protein kinase; p-Akt, phosphorylated thymoma viral proto-oncogene; PBS, phosphate-buffered saline; PD, Parkinson's disease; PD98059, 2-(2-amino-3-methoxyphenyl)-4H-1-benzopyran-4-one; p-ERK, phosphorylated extracellular signal-regulated kinase; PI3K, phosphatidylinositol-3-kinase; p-p70S6K, phosphorylated p70 ribosomal S6 protein kinase; SB-2777011-A, trans- $N$-4-2-(6-cyano-1,2,3,4-tetrahydroisoquinolin-2-yl)ethylcyclohexyl-4quinolinecarboxamide; SCH23390, $(R)-(+)$-7-chloro-8-hydroxy-3-methyl-1-phenyl-2,3,4,5-tetrahydro-1H-3-benzazepine hydrochloride; SN, substantia nigra; TBST, Tris-buffered saline/Tween 20; TH, tyrosine hydroxylase; VTA, ventral tegmental area. 
studies was dedicated to the effect of nicotine on the mesencephalic DAergic system, comparatively little research has focused on morphologic remodeling and structural plasticity. Of interest, morphologic remodeling was found with other addictive drugs, including psychostimulants (Nestler et al., 1993; Mueller et al., 2006; Sarti et al., 2007). Using primary culture from mouse embryo, we recently showed that exposure to amphetamine and cocaine produced structural plasticity in vitro by increasing dendrite number, maximal dendrite length, and soma area of mesencephalic DAergic neurons (Collo et al., 2008,2012 ). These effects were conditional on functional dopamine D3 receptor (D3R), a 7-transmembrane receptor (Missale et al., 1998) implicated in the reinforcing properties of psychostimulants and nicotine (Heidbreder et al., 2005; Le Foll et al., 2007). Of interest, similar structural plasticity was produced by D3R-preferential DAergic agonists (Van Kampen and Eckman, 2006; Collo et al., 2008), agents currently used for PD treatment (Joyce and Millan, 2007).

The evidence for neurotrophic effects of nicotine is not unprecedented. Nicotine exposure attenuates the damage produced by nigrostriatal pathway lesions and fosters regeneration of the DAergic system (Janson et al., 1988a,b; Quik et al., 2006). More recently, transcriptional profiling in VTA showed that nicotine regulates 47 genes involved in neuronal morphogenesis (Doura et al., 2010). Nicotine exerts its effects by binding to the nicotinic acetylcholine receptors (nAChRs), transmembrane pentameric oligomers consisting of different combinations of $9 \alpha$-subunits and $3 \beta$-subunits (Changeux, 2010). In DAergic neurons, the main functional receptors are $\alpha 4 \beta 2$ $\mathrm{nAChRs}$ that play a primary role in controlling DA release, neuronal firing, and nicotine-induced behavior (Picciotto et al., 1998; Marubio et al., 2003; Changeux, 2010).

In this article, we investigated the structural remodeling effects of nicotine in mesencephalic DAergic neurons by studying the morphologic changes produced by nicotine in an established protocol of primary neuronal cultures from mouse embryo (Collo et al., 2008, 2012). Pharmacologic antagonists and neuronal cultures derived from mice carrying $\alpha 4 \mathrm{nAChR}$ subunit or D3R gene knockout (KO) mutation were used to dissect the critical components of structural plasticity produced by nicotine. Because a large body of evidence involves phosphorylation of extracellular signal-regulated kinase (ERK) and thymoma viral proto-oncogene (Akt) in dendrite outgrowth and soma size increase via activation of the mammalian target of rapamycin (mTOR) (Kwon et al., 2003; Jaworski et al., 2005; Kumar et al., 2005), we extended our exploration of nicotine's role to these intracellular pathways. To confirm the relevance in vivo of these observations, we studied the morphologic effects of nicotine on the DAergic system of newborn mice exposed in utero at the same embryonic stage used for the in vitro cultures.

\section{Materials and Methods}

Chemicals. (-)-Nicotine ditartrate, mecamylamine, dihydro$\beta$-erythroidine $(\mathrm{DH} \beta \mathrm{E})$, methyllycaconitine (MLA), 7-hydroxy- $N, N$ di-propyl-2-aminotetralin (7-OH-DPAT), sulpiride, $(R)-(+)-7$-chloro-8hydroxy-3-methyl-1-phenyl-2,3,4,5-tetrahydro- $1 H$-3-benzazepine hydrochloride (SCH23390), rapamycin, 2-(4-morpholinyl)-8-phenyl4H-1-benzopyran-4-one hydrochloride (LY294002), and 2-(2-amino-3methoxyphenyl)-4H-1-benzopyran-4-one (PD98059) were purchased from Tocris Bioscience (Bristol, UK); brain-derived neurotrophic factor (BDNF) was from Alomone Laboratories Ltd. (Jerusalem, Israel); and
SB-277011-A was a gift from C. Heidbreder, GlaxoSmithKline (Verona, Italy). Nicotine, mecamylamine, DH $\beta$ E, MLA, 7-OH-DPAT, SCH23390, and BDNF were dissolved in water; SB-277011-A, rapamycin, PD98059, and LY294002 were dissolved in dimethylsulfoxide ( $>1: 3000$ final dilution), while sulpiride was dissolved in ethanol ( $>1: 2000$ final dilution), all as recommended by producer. For each control treatment, the vehicle was prepared with the respective dilution of dimethylsulfoxide or ethanol.

Animals. CD1 mice were obtained from Charles River Laboratories (Calco, Italy). The following knockout mice were used: mice genetically deprived of dopamine D3R (D3KO) from the Jackson Laboratory (Bar Harbor, ME) (B6.129S4-Drd3Tm1Dac/J) (Accili et al., 1996) and mice genetically deprived of $\alpha 4$ subunit of $\mathrm{nAChR}(\alpha 4 \mathrm{KO})$ (Marubio et al., 2003). As control, C57BL6/J singenic mice were used.

Breeding of all mice was performed to achieve timed pregnancy with the accuracy of \pm 0.5 days. The embryonic day (E) was determined by considering the day of insemination (determined by vaginal plug) as day E0.5. Animal care was in accordance with the European Communities Council Directive of November 24, 1986 (86609/EEC), and in line with Italian law.

Primary Mesencephalic Cultures. Primary mesencephalic cultures were prepared as described in Collo at al. (2008). In brief, ventral mesencephalon tissues were dissected from E12.5 mouse embryos (sex unknown) and collected in ice-cold Hanks' buffered salt solution (EuroClone, Milan, Italy). Tissues were transferred in Accumax (Sigma-Aldrich, St. Louis, MO), mechanically dissociated at room temperature, and resuspended in Neurobasal medium [Gibco (Invitrogen), Carlsbad, CA] containing $2 \mathrm{mM}$ glutamine (EuroClone) and B27 supplement [Gibco (Invitrogen)]. Cells were counted, seeded on poly-D-lysine/ laminin- (Sigma-Aldrich) coated coverslides $\left(5 \times 10^{4}\right.$ cells $/ \mathrm{ml})$ or 12 -well plates $\left(5 \times 10^{5}\right.$ cells $\left./ \mathrm{ml}\right)$, and cultured at $37^{\circ} \mathrm{C}$ in a humidified atmosphere of $5 \% \mathrm{CO}_{2}$ and $95 \%$ air. No fetal bovine serum was added to the cultures; $50 \%$ of the medium was changed every two days until treatment. Under these conditions, the cultures contained $>99.5 \%$ neurons and $<0.5 \%$ astrocytes, as assessed by microtubuleassociated protein 2 and glial fibrillary acidic protein immunostaining, respectively (unpublished data). Pharmacological treatments were conducted in vitro after 5 days from seeding, time required for primary culture stabilization.

In Vivo Prenatal Nicotine Treatment. Pregnant D3KO and singenic wild-type mice were individually housed in a climate-controlled room on a 12/12-hour light/dark cycle with ad libitum access to food and water. Treatments were performed during the light period (9:00 AM6:00 PM). All mice were weighed daily starting at day 10. At day 12.5, pregnant mice weighing $25-30 \mathrm{~g}$ were administered intraperitoneally with two daily doses of $5 \mathrm{mg} / \mathrm{kg}$ nicotine or saline as described by Vezina et al. (2007). Treatments were repeated for 5 days, from E12.5 through E17. After each injection, animals were placed for 30 minutes in a clean cage and inspected for locomotor activity. After delivery, P1 newborn mice (sex unknown) were sacrificed, and the brains were removed and processed for immunohistochemistry.

Immunocytochemistry and Immunofluorescence in Primary Cultures. Immunocytochemistry and immunofluorescence were performed as described in Collo et al. (2012). In brief, for immunocytochemistry, mesencephalic neurons were fixed in phosphatebuffered saline (PBS) containing 3\% paraformaldehyde $3 \%$ sucrose (both Sigma-Aldrich); blocked in PBS containing 0.3\% Triton (Promega, Madison, WI), 1\% normal goat serum (Jackson ImmunoResearch, West Grove, PA), and 5\% bovine serum albumin (BSA; Sigma-Aldrich); and incubated overnight at $4^{\circ} \mathrm{C}$ with a rabbit polyclonal antityrosine hydroxylase (TH) antibody (1:500; Santa Cruz Biotechnology, Santa Cruz, CA), followed by a biotinylated goat anti-rabbit antibody (Jackson ImmunoResearch; 30 minutes at room temperature) and a final incubation with avidin-biotinylated horseradish peroxidase complex (ABC; Vector Laboratories, Burlingame, CA). Color development was achieved by addition of 3,3'diaminobenzidine (SigmaAldrich) for 45 seconds. To study the expression of phosphorylated ERK (p-ERK1/2) or Akt (p-Akt $\left.{ }^{(\text {Thr308) }}\right)$ in dopaminergic neurons, 
mesencephalic cultures were fixed with methanol and incubated with a rabbit polyclonal antibody anti-TH (1:800; Santa Cruz Biotechnology) or a mouse monoclonal antibody anti-TH (1:700; Millipore, Billerica, MA), together with a mouse monoclonal antibody to pERK1/2 (1:400; Cell Signaling Technology, Beverly, MA) or a rabbit polyclonal antibody to p-Akt ${ }^{\text {Thr308) }}$ (1:400; Cell Signaling Technology), respectively. To investigate the expression of Thr-389 phosphorylated p70 ribosomal $\mathrm{S} 6$ protein kinase (p-p70S6K), a mouse monoclonal antibody anti- p-p70S6K (1:500; Cell Signaling Technology) was used together with the rabbit polyclonal antibody anti-TH. Alexa Fluor 488- and Cy3-conjugated secondary antibodies (Jackson ImmunoResearch) were used. As negative control, cultured neurons were incubated with the secondary antibodies only. Each experiment was repeated three times, and all dopaminergic neurons present in two coverslides for each treatment condition were assessed. Immunofluorescences for $\mathrm{p}-\mathrm{ERK} 1 / 2$ and $\mathrm{p}-\mathrm{Akt}^{(\mathrm{Thr} 308)}$ were visualized using an Olympus IX51 microscope (Hamburg, Germany), and TH- immunoreactivity (IR) neurons immunostained for p-p70S6K were analyzed using a Zeiss LSM 510 Meta confocal microscope equipped with PlanApochromat $63 \times / 1.4$ numerical aperture oil objective and LSM 510 Meta Software, version 3.5 (Carl Zeiss AG, Oberkochen, Germany). Confocal digital images were further examined using the Axio Vision Release, version 4.8.2, software (Carl Zeiss AG).

Immunohistochemistry of Brain Sections. Newborn mice were sacrificed at $\mathrm{P} 1$, and brain was removed, fixed overnight in PBS $4 \%$ paraformaldehyde, cryoprotected in $20 \%$ sucrose, and rapidly frozen by immersion in isopentane on dry ice. For each brain, a complete set of coronal sections was cut through the SN and VTA at $30 \mu \mathrm{m}$. Sections were mounted on slides, blocked, and permeabilized with PBS, 5\% BSA (Sigma-Aldrich), and 0.1\% Triton (Sigma-Aldrich) for 30 minutes at room temperature. They were incubated with the rabbit anti-TH antibody (1:500; Santa Cruz Biotechnology) diluted in PBS and $1 \% \mathrm{BSA}$ overnight at $4^{\circ} \mathrm{C}$. Then, they were washed and incubated with a biotinylated goat anti-rabbit antibody (Jackson ImmunoResearch) diluted in PBS and 1\% BSA for 30 minutes at room temperature, followed by the ABComplex and development with 3,3'-diaminobenzidine (DAB).

Computer-Assisted Morphologic Analysis. Digital images from primary neuronal cultures or mesencephalic brain tissues were acquired with an Olympus IX51 microscope connected to an Olympus digital camera and a PC. Morphometric measurements were performed by a blinded examiner on digitalized images using Image-Pro Plus software (Media Cybernetics, Bethesda, MD). For in vitro primary neuronal culture, the morphologic indicators of structural plasticity were (i) the maximal dendrite length, (ii) the number of primary dendrites, and (iii) the soma area (Schmidt et al., 1996). Maximal dendrite length was defined as the distance from the soma (hillock base) to the tip of the longest dendrite for each neuron; dendrites shorter than $20 \mu \mathrm{m}$ were excluded from the analysis. Primary dendrites were defined as those directly stemming from the soma. Soma area was assessed by measuring the surface $\left(\mu \mathrm{m}^{2}\right)$ included by the external perimeter drawn on the cell membrane of neurons identified by TH-IR staining. Two coverslides per treatment group were examined to obtain measurements from at least 30 neurons. For ex vivo studies, computerassisted morphometry was performed as described in Collo et al. (2012). The effects of nicotine on newborn mice were assessed by measuring in each brain the soma area of 90-120 dopaminergic neurons at various rostrocaudal levels of SN and VTA, respectively. The same rostrocaudal levels were considered in each treatment group to keep anatomic variance partially controlled. In a typical experiment, eight newborn mice from three different mothers per group were included. The soma area of dopaminergic neurons on histologic preparations was measured as described for TH-IR primary neurons in cultures.

Western Blotting. Western blotting was performed at different time points (1-10 minutes) after challenge with nicotine $(10 \mu \mathrm{M})$ and/ or after pretreatments with either D3R antagonist SB-277011-A (50 nM) or phosphatidylinositol-3-kinase (PI3K) antagonist LY294002 $(10 \mu \mathrm{M})$. For studies in D3KO and singenic wild-type mice, challenges were produced with nicotine $(10 \mu \mathrm{M})$ and 7-OH-DPAT $(50 \mathrm{nM})$. Mesencephalic cultures were washed with ice-cold PBS and lysed in a buffer containing $50 \mathrm{mM}$ Tris ( $\mathrm{pH} 7.4$ ), $150 \mathrm{mM} \mathrm{NaCl}, 0.5 \%$ sodium deoxycholate, $0.1 \%$ sodium dodecyl sulfate, $1 \%$ Igepal, $1 \mathrm{mM}$ polymethanesulphonyl fluoride, and complete protease inhibitors (Roche Diagnostics, Mannhein, Germany) for 10 minutes in ice. Neurons were homogenized with a probe-type sonicator on ice and centrifuged at $13,000 \mathrm{~g}$ for 10 minutes at $4^{\circ} \mathrm{C}$. The protein concentration was measured using a DC-protein assay (Bio-Rad, Hercules, CA). Twenty micrograms of total proteins were resolved by $10 \%$ sodium dodecyl sulfate polyacrylamide gel electrophoresis and blotted onto a polyvinylidene difluoride membrane (Immobilon-P; Millipore). Membranes were blocked in Tris-buffered saline/Tween 20 (TBST; $25 \mathrm{mM}$ Tris base, $137 \mathrm{mM} \mathrm{NaCl}, 2.7 \mathrm{mM} \mathrm{KCl}$ and $0.1 \%$ Tween-20, $\mathrm{pH} \mathrm{7.6)}$ and $5 \%$ dry milk and incubated overnight with the primary antibody diluted in 5\% BSA in TBST. The primary antibodies used were antip-ERK1/2 (1:1000) and anti-ERK1/2 (1:1000) mouse monoclonal antibodies (both from Santa Cruz Biotechnology); anti-p-Akt ${ }^{\text {(Thr308) }}$ (1:2000) rabbit polyclonal antibody; anti-Akt (1:1000) rabbit polyclonal antibody; anti-p-p70S6K (1:2000) and anti-p70S6K (1:2000) mouse monoclonal antibodies (all from Cell Signaling Technology); anti-TH (1:1000) rabbit polyclonal antibody (Millipore); and anti$\alpha$-Tubulin (1:20000) mouse monoclonal antibody (Sigma-Aldrich). In a typical experiment, the same membrane was processed in the following order: incubation with anti-p-ERK1/2 antibody, stripping with the Re-Blot Plus Strong Solution (Millipore), incubation with anti-ERK1/2 antibody, incubation with anti-TH antibody, and incubation with anti- $\alpha$-Tubulin antibody. An equivalent protocol was performed for p-Akt and p-p70S6K blotting. After the incubation with primary antibodies, blots were washed in TBST, incubated with appropriate horseradish peroxidase-conjugated secondary antibodies (goat anti-rabbit or goat anti-mouse; Santa Cruz Biotechnology), and developed using a chemiluminescent substrate (ECL, LiteAblot Extend; EuroClone). Specific bands were analyzed by densitometric scanning of exposed film using Gel-pro analyzer software (Media Cybernetics). In each experiment, the specific signal of p-ERK1/2, $\mathrm{p}$-Akt, and p-p70S6K proteins was normalized to the corresponding ERK1/2, Akt, or p70S6K signals, respectively, and then to the level of TH and $\alpha$-Tubulin measured in the same preparation.

Statistical Analysis. Data were expressed as mean \pm S.E.M. if not stated otherwise. Significant differences from control conditions were determined using either one-way or two-way analysis of variance, followed by a posteriori Bonferroni's test for multiple comparisons provided by GraphPad Prism, version 4.0 for Windows (GraphPad Software, San Diego, CA).

\section{Results}

Nicotine-Induced Structural Plasticity in Primary Cultures of Mesencephalic DAergic Neurons. Mesencephalic DAergic neurons were visualized using a TH antibody as shown in Fig. 1, A and B. The time- and dose-dependency of nicotine effects on structural plasticity measured as maximal dendrite length, number of primary dendrites, and cell body size are shown in Fig. 1, C-E. Various doses of nicotine $(0.1,1$, and $10 \mu \mathrm{M}$ ) or vehicle were used, and their effects on neuronal cultures were seen at two different time points: 24 hours and 72 hours. The maximal length of dendrites showed a significant treatment effect $[F(3,232)=54.32 ; P<0.0001]$, with significant difference from vehicle already at $1 \mu \mathrm{M}$ nicotine and stronger effect at $10 \mu \mathrm{M}$ nicotine (Bonferroni's test $P<$ 0.001 versus vehicle). This effect was more marked at 72 hours, as indicated by the significant time effect $[F(1,232)=$ 9.28; $P<0.001$ ], while the treatment $\times$ time interaction was not significant (Fig. 1C). The analysis of number of primary dendrites indicated a dose-related treatment effect 


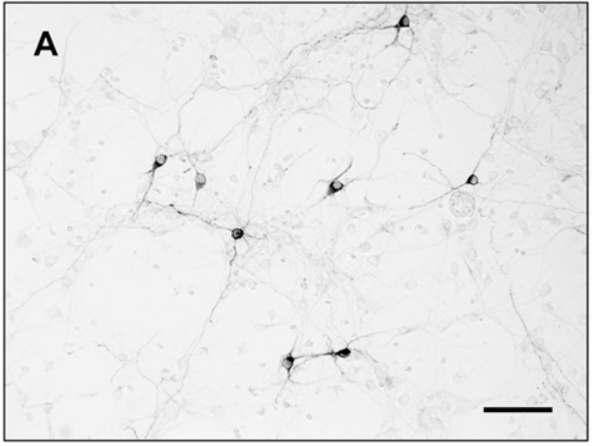

C

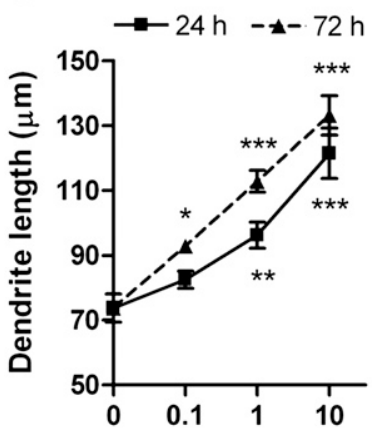

D

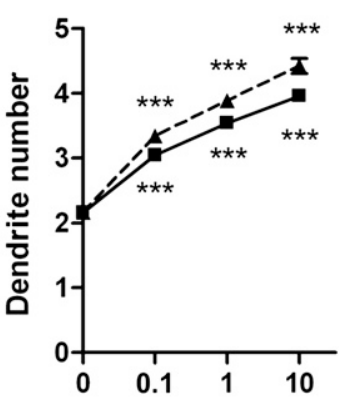

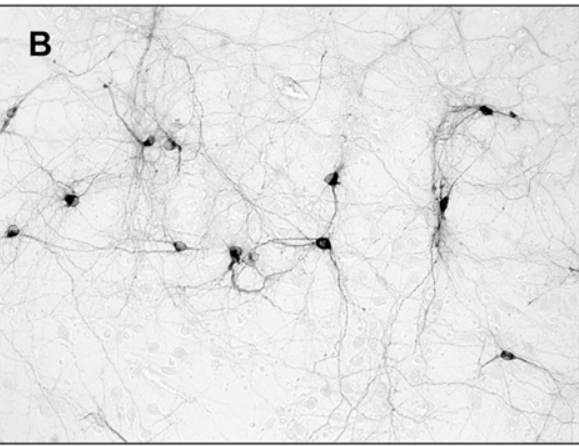

E

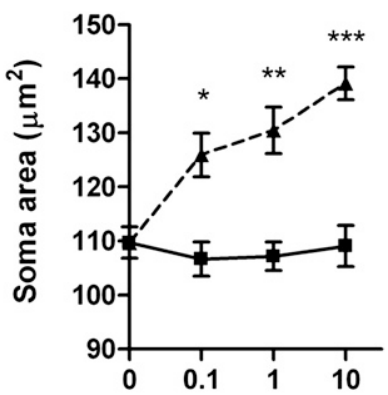

Fig. 1. Morphologic effects of nicotine on mesencephalic dopaminergic neurons. (A and B) Representative photomicrographs of TH-IR neurons after 72 hours exposure to vehicle (A) or $10 \mu \mathrm{M}$ nicotine (B). Scale bar: $60 \mu \mathrm{m}$. (C-E) Dose-response curve and time-dependence of morphologic effects of nicotine on maximal dendrite length $(\mathrm{C})$, number of primary dendrites (D), and soma area (E) measured 24 hours (continuous line) or 72 hours (dotted line) after treatment $(0=$ vehicle $)$. In all panels, values are represented as mean \pm S.E.M. $\left({ }^{* * *} P<0.001 ; * * P<0.01 ; * P<0.05\right.$ versus vehicle, post-hoc Bonferroni's test).

Nicotine $(\mu \mathrm{M})$

$[F(3,344)=141.7, P<0.0001]$, with significant differences between each dose (Bonferroni's test $P<0.001$ ) and a significant time effect $[F(1,344)=17.53 ; P<0.0001]$ with the strongest signal at 72 hours (Bonferroni's test $P<0.01$ ). Again, the treatment $\times$ time interaction was not significant. The analysis of the cell soma area showed significant treatment effect $[F(3,232)=6.19 ; P<0.0005]$ and time effect $[F(1,232)=57.01$; $P<0.0001$ ], with a treatment $\times$ time interaction that was highly significant $[F(1,3)=7.20 ; P<0.0001]$. Post-hoc analysis showed that lack of effects at 24 hours versus baseline was associated with a strong growth at 72 hours, an effect that showed clear dose-dependency (Fig. 1E). In one independent series of experiments, when the dose of $10 \mu \mathrm{M}$ nicotine was incubated for 10 minutes, 1 hour, 6 hours, 12 hours, 24 hours, and 72 hours, significant difference from vehicle of dendrite length, number, and soma area were observed starting at 12 hours, progressively increasing with a peak at 72 hours (unpublished data). On the basis of these observations, the nicotine dose of $10 \mu \mathrm{M}$ and an incubation time of 72 hours were chosen for further studies.

Nicotine-Induced Structural Plasticity Depends on $\alpha 4 \beta 2^{*}$ nAChRs: Pharmacological and KO Mouse Studies. The involvement of $\mathrm{nAChRs}$ in nicotine-induced structural plasticity of mesencephalic DAergic neurons was tested in independent experiments after pretreatments with the nonselective nAChR antagonist mecamylamine (MEC), the selective $\beta 2^{*} \mathrm{nAChR}$ antagonist $\mathrm{DH} \beta \mathrm{E}$, and the selective $\alpha 7$ nAChR antagonist MLA (Fig. 2, A-C). The maximal dendrite length showed a significant treatment effect $[F(7,232)=$ 17.58; $P<0.0001$ ], with nicotine producing a significant increase (Bonferroni's test $P<0.001$ ), and this effect was significantly attenuated by MEC or $\mathrm{DH} \beta \mathrm{E}$ (Bonferroni's test $P<0.01$ ), but not by MLA; incubation with nAChR antagonists alone (i.e., when vehicle was added instead of nicotine) showed no difference from vehicle (Fig. 2A). Similar significant effects of treatment were observed for the number of primary dendrites $[F(7,471)=9.69 ; P<0.0001]$ (Fig. 2B), and for the soma area $[F(7,392)=12.66 ; P<0.0001]$ (Fig. 2C), the increase produced by nicotine (Bonferroni's test $P<0.001$ ) was significantly attenuated by MEC or $\mathrm{DH} \beta \mathrm{E}$ (Bonferroni's test $P<0.01$ ) but not by MLA, whereas no difference was observed when the nAChR antagonists were given alone. Overall, these data indicate the involvement of $\beta 2^{*} \mathrm{nAChR}$ and excluded the involvement of $\alpha 7^{*} \mathrm{nAChR}$.

Because the most common $\beta 2 * \mathrm{nAChR}$ expressed in DAergic neurons is the $\alpha 4 \beta 2^{*}$ nAChR (Champtiaux et al., 2003; ZhaoShea et al., 2011), we extended these observations with use of cultures of mesencephalic neurons obtained from $\alpha 4 \mathrm{nAChR}$ $\mathrm{KO}(\alpha 4 \mathrm{KO})$ mice. Structural plasticity was tested by adding nicotine $(10 \mu \mathrm{M})$, 7-OH-DPAT ( $5 \mathrm{nM})$, and BDNF $(10 \mathrm{ng} / \mathrm{ml})$ to the incubation media 72 hours before morphologic assessment. The D3R agonist 7-OH-DPAT $(5 \mathrm{nM})$ was included because we previously showed a reliable neurotrophic-like effect in the same mesencephalic preparation (Collo et al., 2008), whereas BDNF is a well-known neurotrophic factor for DAergic neurons (Hyman et al., 1991). In this experiment, cultures from $\alpha 4 \mathrm{KO}$ mice were compared with cultures from wild-type mice. Maximal dendrite length of DAergic neurons isolated from $\alpha 4 \mathrm{KO}$ mouse embryos was significantly increased by BDNF and by 7-OH-DPAT (Bonferroni's test $P<$ 0.001 ), but not by nicotine, at variance with what was obtained in wild-type mice that responded significantly to all treatments (Bonferroni's test $P<0.01$; or $P<0.001$ ) (Fig. 2D).

The number of primary dendrites in response to treatments was also significantly affected by the $\alpha 4 \mathrm{KO}$ genotype (Fig. 2E). Cultures prepared from $\alpha 4 \mathrm{KO}$ mice responded significantly to 7-OH-DPAT and BDNF (Bonferroni's test $P<0.05$; or $P<$ 0.01 ) but were not affected by nicotine, whereas in cultures 

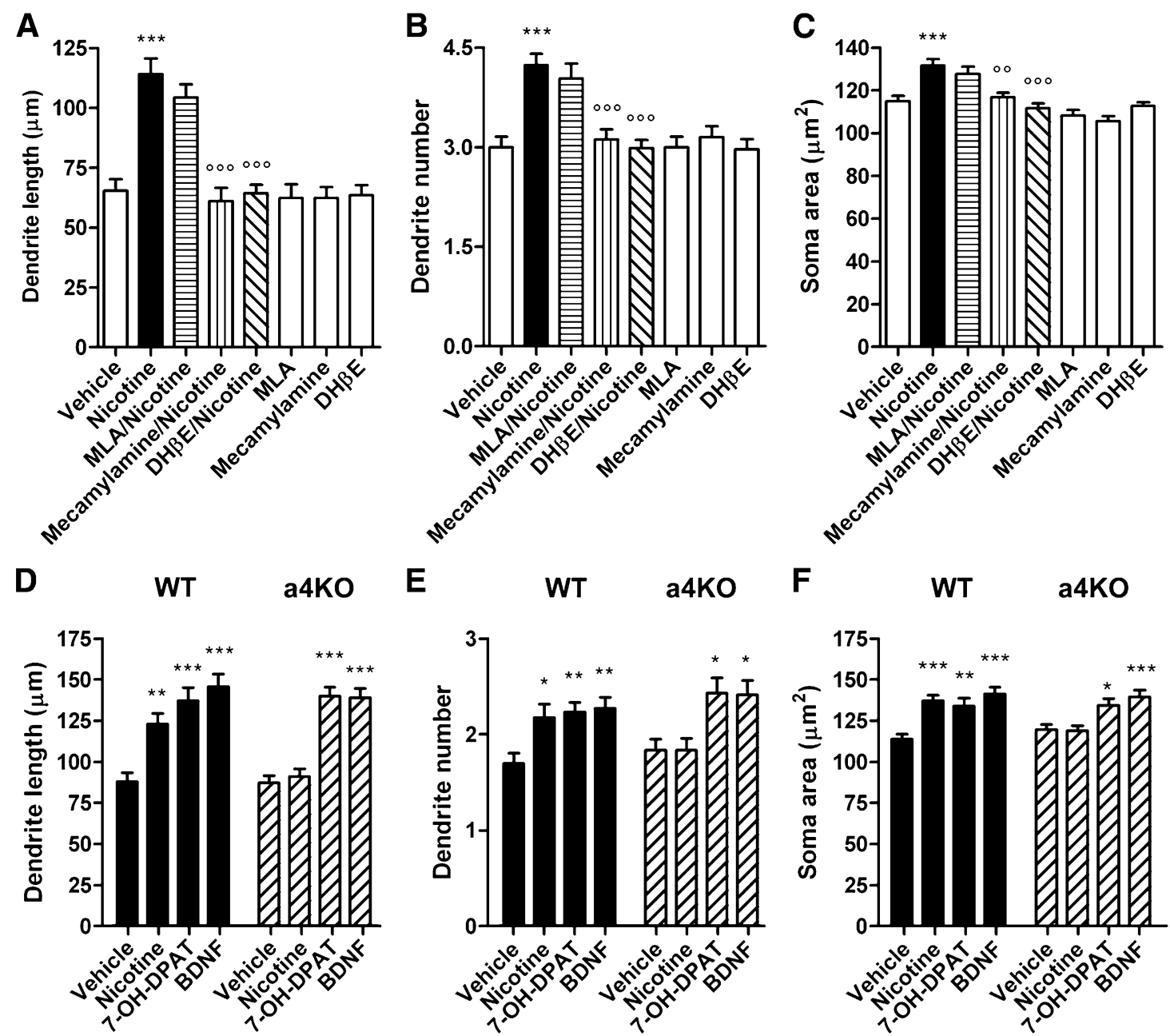

Fig. 2. Involvement of nicotinic acetylcholine receptor subtypes in morphologic effects of nicotine on mesencephalic dopaminergic neurons studied 72 hours after treatment. (A-C) Representative experiment of the effects of nAChR antagonists methyllycaconitine (200 nM), mecamylamine (100 $\mu \mathrm{M})$, dihydro- $\beta$-erytroidine $(10 \mu \mathrm{M})$, or vehicle added 20 minutes before nicotine $(10 \mu \mathrm{M})$ on maximal dendrite length, number of primary dendrites, and soma area. (D-F) Morphologic effects of nicotine on TH-IR neurons from wild-type and $\alpha 4 \mathrm{KO}$ mice 72 hours after treatment expressed as mean values. Mesencephalic neurons were exposed to nicotine $(10 \mu \mathrm{M})$, 7-OH-DPAT ( $5 \mathrm{nM})$, or BDNF $(10 \mathrm{ng} / \mathrm{ml})$, and the following end points were measured: maximal dendrite length (D), number of primary dendrites (E), and soma area (F). Experiments were replicated three times. Values are represented as mean \pm S.E.M. $\left({ }^{* * *} P<0.001\right.$ versus vehicle; ${ }^{* *} P<0.01$ versus vehicle; ${ }^{*} P<0.05$ versus vehicle; ${ }^{000} P<0.001$ versus nicotine; ${ }^{\circ 0} P<0.01$ versus nicotine; post-hoc Bonferroni's test).

prepared from wild-type mice, all treatments were effective (Bonferroni's test $P<0.05$; or $P<0.01$ ). Similar results were obtained when measuring the soma areas (Fig. 2F).

Overall, this study indicates that neurons carrying $\alpha 4$ nAChR null mutation are still capable of responding to neurotrophic stimuli, but not to nicotine, indicating a critical role of the $\alpha 4 \beta 2 * \mathrm{nAChR}$ in the structural plasticity produced by nicotine.

Nicotine-Induced Structural Plasticity Depends on Dopaminergic D3R: Pharmacological and D3KO Mouse Studies. To test this hypothesis, the involvement of dopamine receptors in nicotine-induced structural plasticity of mesencephalic dopaminergic neurons was assessed using pretreatments with the D1R antagonist SCH23390 (1 $\mu \mathrm{M})$, the D2D3R antagonist sulpiride $(5 \mu \mathrm{M})$, or the D3R antagonist SB-277011-A (50 $\mathrm{nM})$ added to the cultures 20 minutes before nicotine $(10 \mu \mathrm{M})$ and studied after 72 hours. The maximal dendrite length was significantly affected by the treatment $[F(7,279)=13.29 ; P<0.0001]$, with the nicotineinduced increase (Bonferroni's test $P<0.001$ ) significantly attenuated by sulpiride (Bonferroni's test $P<0.01$ ) and by SB-277011-A (Bonferroni's test $P<0.001$ ), but not by SCH23390, whereas incubation with dopamine antagonists alone, when vehicle was added instead of nicotine, showed no difference (Fig. 3A). Analogous results were obtained by measuring the number of primary dendrites, with a significant treatment effect $[F(7,383)=10.28 ; P<0.0001]$; the nicotineinduced increase (Bonferroni's test $P<0.001$ ) was attenuated by sulpiride (Bonferroni's test $P<0.001$ ) or SB-277011-A (Bonferroni's test $P<0.001$ ), but not by SCH23390 (Fig. 3B). The soma area was also affected by the treatments $[F(7,399)=$ 9.79; $P<0.0001]$ in the same way described for maximal dendrite length and number of primary dendrites (Fig. 3C). These data support a critical role for D3R in mediating the nicotine effects on dopaminergic neuron structure.

The critical role of D3 receptors suggested by these pharmacologic observations was confirmed using mesencephalic neurons obtained from D3KO mice and compared with primary neurons from singenic wild-type mice. Structural plasticity was assessed 72 hours after incubation with nicotine 

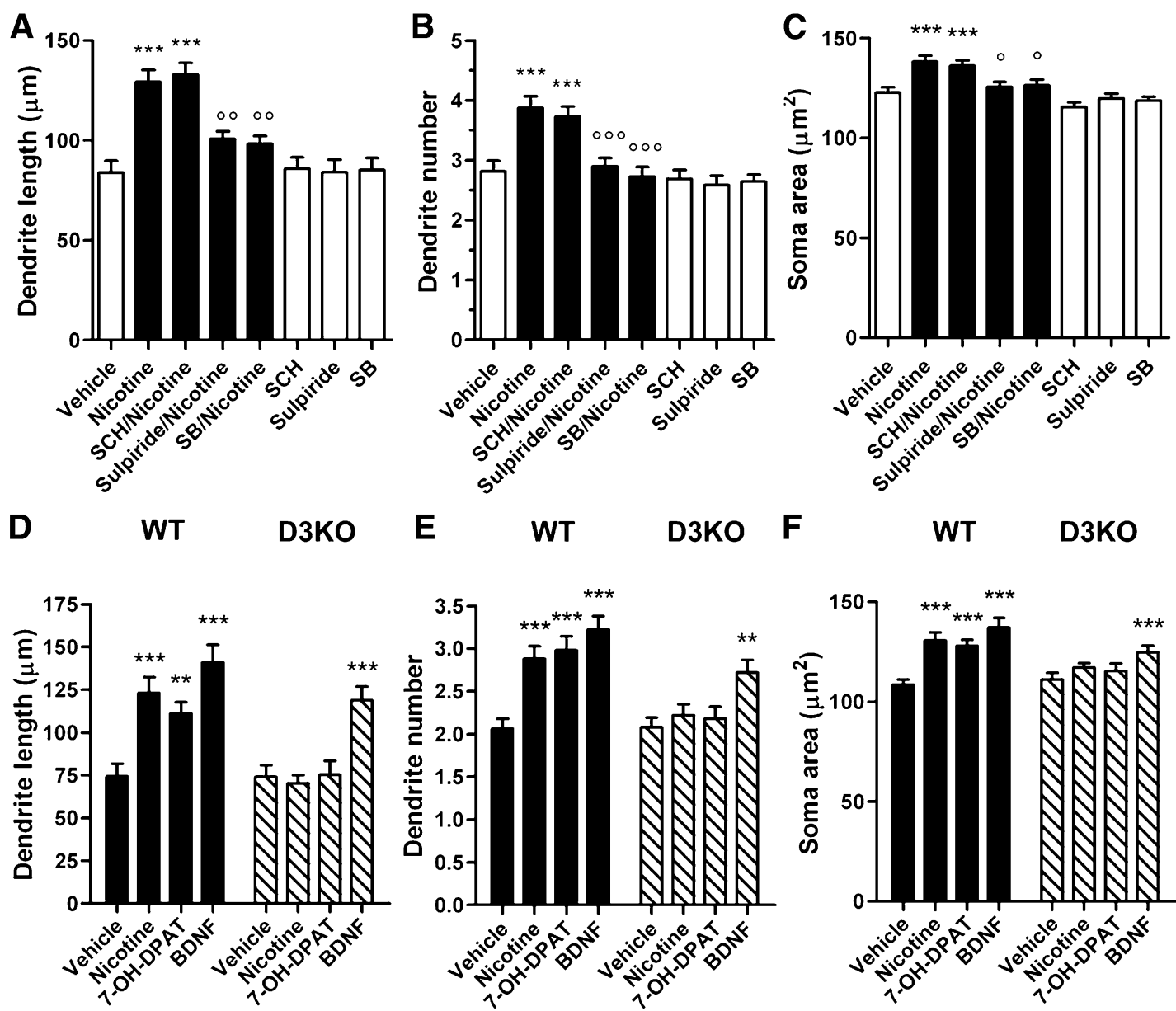

WT

D3KO

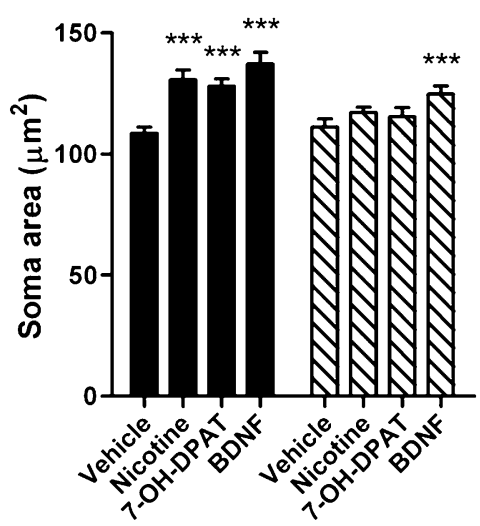

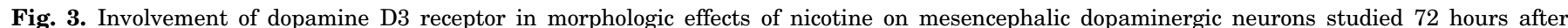

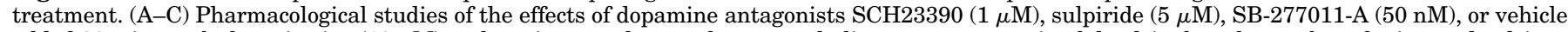

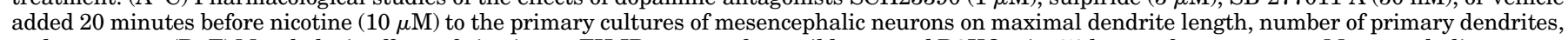

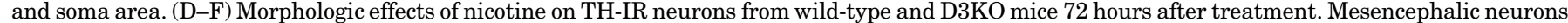

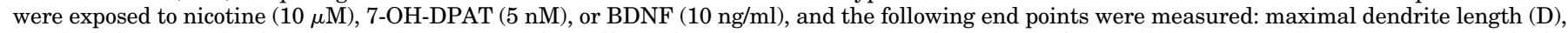

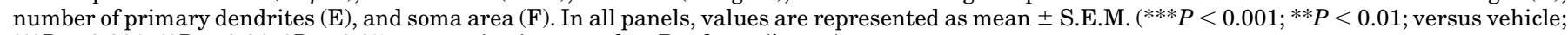
${ }^{\circ \circ} \mathrm{P}<0.001 ;{ }^{\circ} \mathrm{P}<0.01 ;{ }^{\circ} \mathrm{P}<0.05$ versus nicotine; post-hoc Bonferroni's test).

(10 $\mu \mathrm{M})$, the D3R agonist 7-OH-DPAT ( $5 \mathrm{nM})$, the neurotrophic factor BDNF (10 ng/ml), or vehicle. Maximal dendrite length was significantly affected by genotype, showing that cultures prepared from wild-type mice responded significantly to nicotine, 7-OH-DPAT, or BDNF (Bonferroni's test $P<0.001$ ), whereas cultures prepared from D3KO mice responded significantly only to BDNF (Bonferroni's test $P<0.001$ ), but not to nicotine or 7-OH-DPAT (Fig. 3A). The number of primary dendrites and soma size were also significantly affected by genotype, showing that, in cultures prepared from wild-type mice, nicotine, BDNF, and 7-OH-DPAT increased the number of primary dendrites (Bonferroni's test $P<0.001$ ), whereas cultures prepared from D3KO mice significantly responded only to BDNF (Bonferroni's test $P<0.001$ ), but not to nicotine or 7-OH-DPAT (Fig. 3, B and C). Overall, this study indicates that neurons carrying a D3R null mutation are still capable of responding to neurotrophic stimuli conveyed by BDNF, but not to those of nicotine and 7-OH-DPAT effects, suggesting a necessary role for D3R in mediating nicotine effects.

Nicotine Exposure in Pregnant Mothers Produced Structural Plasticity in Mesencephalic Neurons of the Offspring: Lack of the Effects in D3KO Mice. The in vivo relevance of the nicotine-induced changes observed in cultured mesencephalic dopaminergic neurons dissected from the E12.5 mouse embryo was investigated by repeatedly exposing mouse embryos to nicotine while in uterus from E12.5 through E17. Treatments were performed in both wildtype and D3KO pregnant mice, and effects on soma area were measured on the mesencephalon of newborns (Fig. 4, A and B). Two-way analysis of variance was used to assess the genotype $\times$ treatment effects and interactions. The analysis of soma area in the VTA showed a significant genotype effect $[F(1,28)=33.23 ; P<0.0001]$, treatment effect $[F(1,28)=25.86$; $P<0.0001]$, and interaction $[F(1,28)=14.44 ; P<0.005]$, with post-hoc test showing effect of nicotine only in wild-type mice $(P<0.001)$ (Fig. 4C). Soma area in the $\mathrm{SN}$ showed a significant genotype effect $[F(1,28)=29.42 ; P<0.0001]$, treatment effect $[F(1,28)=19.50 ; P<0.0005]$, and interaction $[F(1,28)=19.40$; $P<0.001$, with post-hoc test showing effect of nicotine only in wild-type mice $(P<0.001)$ (Fig. $4 \mathrm{D})$.

Nicotine-Induced Activation of the Intracellular ERK and Akt-mTOR Pathways in Primary Dopaminergic Neurons Is Mediated by D3R. The effect of nicotine on ERK1/2 phosphorylation was investigated by challenging 

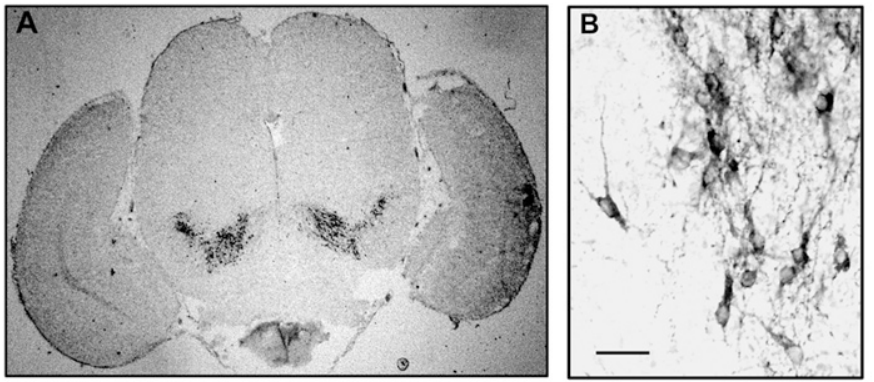

C
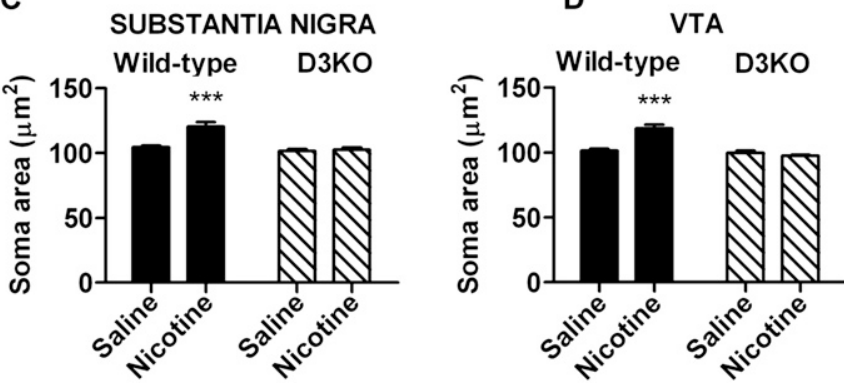

Fig. 4. Effects of prenatal nicotine exposure on structural plasticity of dopaminergic neurons of wild-type and D3KO newborn mice. (A) Representative low-magnification photomicrograph of a coronal brain section of a P1 mouse exposed to daily treatment with nicotine intraperitoneally ( $5 \mathrm{mg} / \mathrm{kg} / \mathrm{die})$ in utero from E12.5 through E17. Sections were immunostained with anti-TH antibody to identify dopaminergic neurons in VTA and SN. (B) High magnification of TH-IR dopaminergic neurons from VTA, in which soma area was investigated. (C and D) Nicotine effects on the soma area of TH-IR neurons in the VTA and SN, respectively, of wild-type and D3KO mice. In all panels, values are represented as mean \pm S.E.M. ( $* * * P<0.001$ versus saline; post-hoc Bonferroni's test). Scale bar: $60 \mu \mathrm{m}$.

mesencephalic neurons in culture with $10 \mu \mathrm{M}$ nicotine. Western blot analysis over time showed an increase of p-ERK1/2 $[F(4,23)=7.63 ; P<0.001]$, with a significant peak activity versus vehicle at 2 minutes (Bonferroni's test $P<$ 0.01) (Fig. 5A). Incubation with SB-277011-A (50 nM) 20 minutes before challenge significantly attenuated the p-ERK1/2 peak increase $[F(3,15)=8.91 ; P<0.005]$, providing evidence of D3R involvement. No effect on $\mathrm{p}$-ERK1/2 was produced by SB-277011-A when tested alone (Fig. 5B). Moreover, 20 minutes pretreatment with the PI3K inhibitor LY294002 $(10 \mu \mathrm{M})$ significantly antagonized the peak increase of $\mathrm{p}-\mathrm{ERK} 1 / 2$ levels $[F(3,27)=12.41 ; P<0.0001]$, whereas no effects on ERK1/2 phosphorylation were observed when LY294002 was tested alone (Fig. 5C). Similar results were obtained in parallel experiments using double-staining immunofluorescence aimed to localize the expression of $\mathrm{p}-\mathrm{ERK} 1 / 2$ in dopaminergic neurons. Nicotine activated p-ERK in TH-IR neurons (Fig. 5, D-F), and its effect was blocked by pretreatment with either SB-277011-A (Fig. 5, G-I) or LY294002 (Fig. 5, M-O). In control experiments, incubation with either SB-277011-A (Fig. 5, J-L) or LY294002 (Fig. 5, P-R) alone did not affect p-ERK1/2 expression.

Mesencephalic neurons obtained from embryos of D3KO and singenic wild-type mice were processed in separated cultures in the same experiment. The effects of either nicotine $(10 \mu \mathrm{M})$ or $7-\mathrm{OH}-\mathrm{DPAT}(50 \mathrm{nM})$ on ERK1/2 phosphorylation were investigated by Western blot analysis 2 minutes after treatment. Nicotine and 7-OH-DPAT significantly increased p-ERK1/2 in wild-type mice $[F(2,24)=5.68 ; P<0.01]$ but not in D3KO mice, as indicated by the highly significant interaction $[F(2,24)=13.60 ; P<0.0001]$ and genotype effect $[F(1,24)=46.40 ; P<0.0001]$ obtained with two-way analysis of variance (Fig. $5 \mathrm{~S}$ ).

The effect of nicotine on Akt phosphorylation was investigated by challenging mesencephalic neurons with $10 \mu \mathrm{M}$ nicotine. Western blot analysis showed a rapid increase in Akt phosphorylation $[F(4,27)=9.03 ; P<0.0002]$, reaching the peak activity at 1 minute exposure (Bonferroni's test $P<0.01$ ) (Fig. 6A). Incubation with SB-277011-A (50 nM) 20 minutes before challenge significantly attenuated the increase of Akt phosphorylation produced by nicotine $[F(3,19)=8.40 ; P<$ 0.002] (Fig. 6B). Moreover, 20 minutes pretreatment with LY294002 $(10 \mu \mathrm{M})$ antagonized the significant increase of pAkt levels $[F(3,19)=7.91 ; P<0.002]$ (Fig. 6C). Similar results were obtained in parallel experiments using double-staining immunofluorescence aimed to localize the expression of p-AktIR in dopaminergic neurons. Nicotine activated p-Akt in TH-IR neurons (Fig. 6, D-F), whereas its effect was blocked by pretreatment with either SB-277011-A (Fig. 6, G-I) or LY294002 (Fig. 6, M-O). In control experiments, incubation with either SB-277011-A (Fig. 6, J-L) or LY294002 (Fig. 6, $\mathrm{P}-\mathrm{R}$ ) alone did not affect p-Akt expression. Mesencephalic neurons obtained from embryos of D3KO and singenic wildtype mice were processed in separated cultures in the same experiment. Increase of p-Akt was produced by nicotine and 7-OH-DPAT in cultures from wild-type mice but not in D3KO mice, as indicated by the highly significant interaction $[F(2,24)=$ 15.73; $P<0.02]$ and genotype effect $[F(1,24)=26.10 ; P<$ 0.0001 ] obtained with two-way analysis of variance (Fig. $6 \mathrm{~S}$ ).

The effect of nicotine on p70S6K phosphorylation, one of the main substrates of the activated mammalian target of rapamycin complex 1 (mTORC1), was investigated by challenging mesencephalic neurons with $10 \mu \mathrm{M}$ nicotine. Western blot analysis showed a delayed increase of p70S6K phosphorylation $[F(4,21)=8.00 ; P<0.001]$ that reached the peak activity at 5 minutes exposure (Bonferroni's test $P<0.001$ ) (Fig. 7A). Incubation with SB-277011-A (50 nM) 20 minutes before challenge significantly attenuated the increase of p70S6K phosphorylation produced by nicotine $[F(3,21)=7.61$; $P<0.002$ ] (Fig. 7B). Moreover, 20 minutes pretreatment with LY294002 $(10 \mu \mathrm{M})$ antagonized the increase of p-p70S6K levels $[F(3,21)=10.03 ; P<0.0005]$ (Fig. 7C).

Information about colocalization of p-p70S6K-IR in TH-IR neurons after nicotine was obtained in parallel experiments using confocal microscopy (Fig. 7, D-L). Nicotine activated pp70S6K-IR in TH-IR neurons (Fig. 7D). Confocal reconstructed TH-IR dendrites visualized with different angles confirmed the presence of p-p70S6K-IR and TH-IR in the same structures (Fig. 7, E and F). Of interest, p-p70S6K-IR and TH-IR showed only a partial overlap of IR, suggesting localization in different dendrite microdomains (Fig. 7, E and F). No p-p70S6K-IR was observed in TH-IR neurons and dendrites when the treatment was vehicle (Fig. 7, G and $\mathrm{H}$ ) or following nicotine after 20 minutes pretreatment with SB277011-A (Fig. 7, I and J) or LY294002 (Fig. 7, K and L), confirming the data obtained with Western blot.

Nicotine-Induced Structural Plasticity Is Blocked by Inhibition of the D3R Intracellular Signaling Pathways. The involvement of phosphokinases that mediate the activation of the ERK1/2, Akt, and mTORC1 intracellular pathways in determining nicotine-induced long term structural 


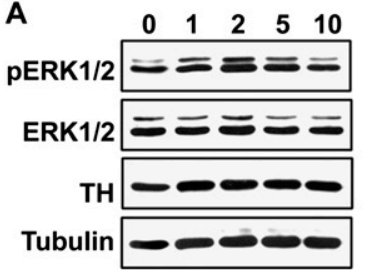

B

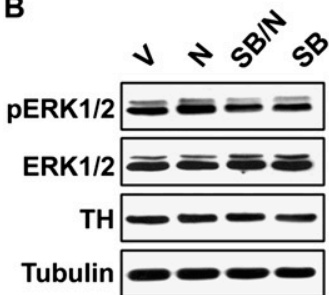

C

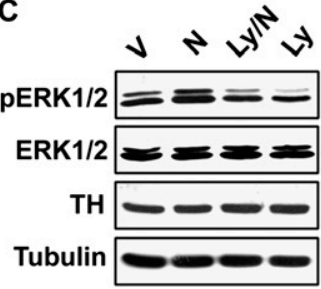

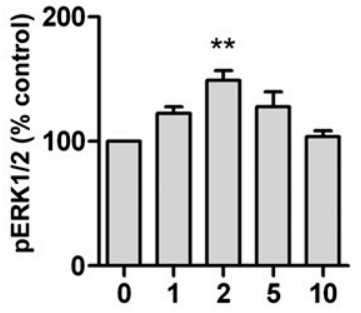
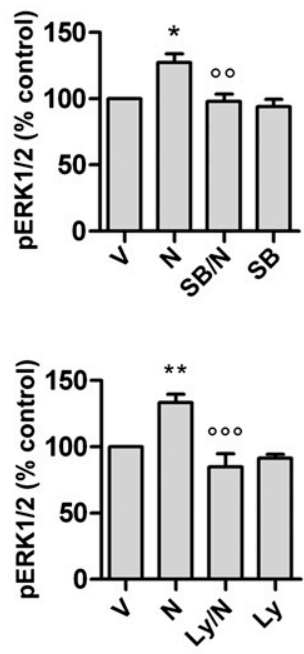

S

WT

D3KO $\checkmark+10$ Pl

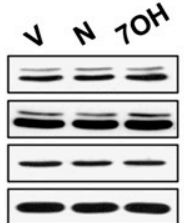

TH
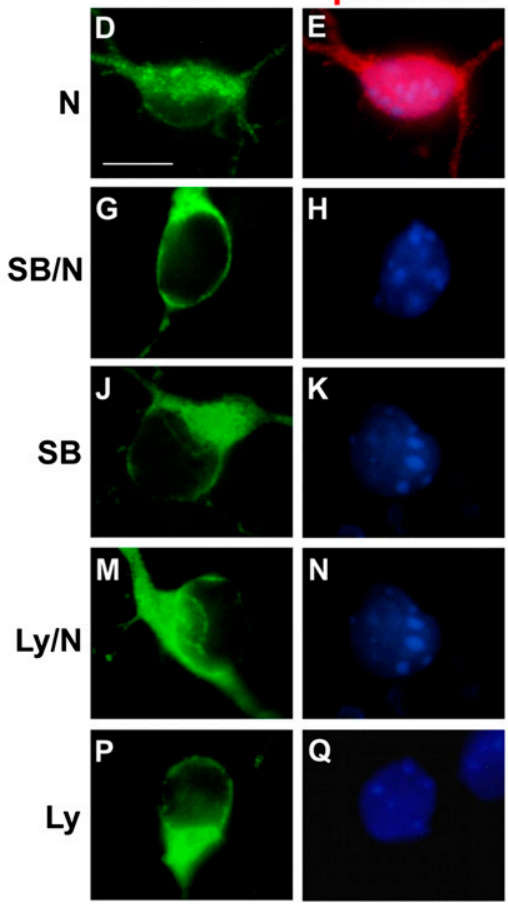

pERK1/2 TH/pERK1/2
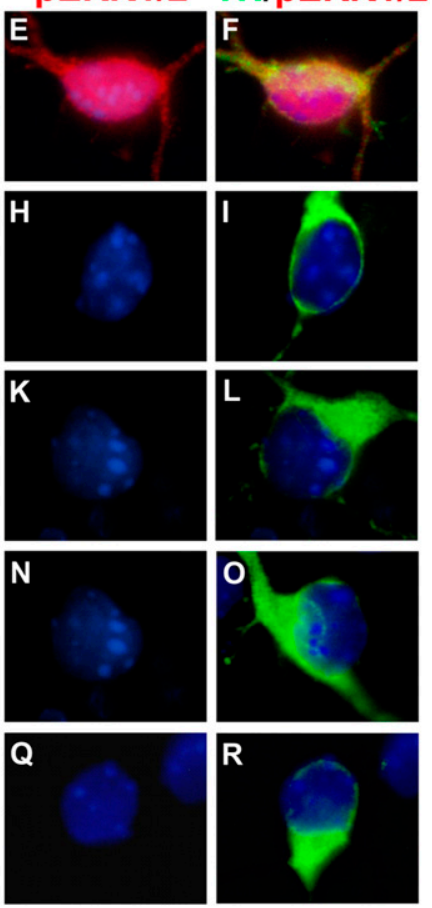
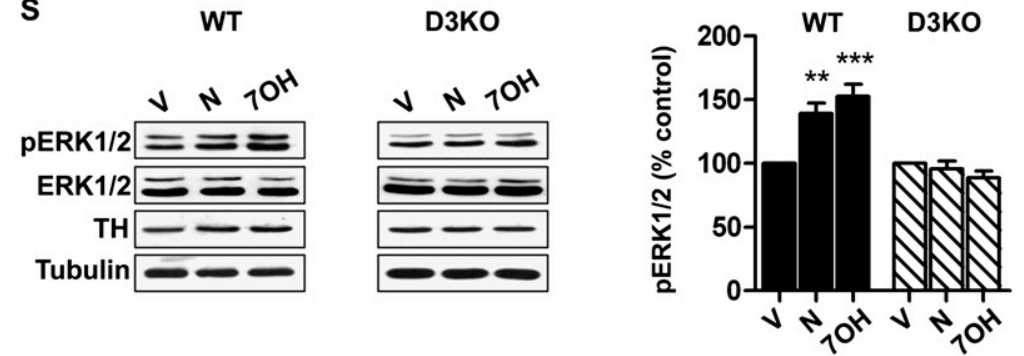

Fig. 5. ERK1/2 phosphorylation activated by nicotine challenge in mesencephalic TH-IR neurons is prevented by D3R antagonist SB-277011-A and PI3K inhibitor LY294002 and is blocked in D3KO mice. (A) Representative time course of ERK1/2 phosphorylation induced by nicotine (10 $\mu$ M) at $0,1,2$ 5 , and 10 minutes; cell lysates were processed for Western blot analysis that was performed using antibodies to detect pERK1/2, ERK1/2, TH, and tubulin levels; membranes were stripped and reprobed as described in Materials and Methods. Right panel, densitometric analysis of blots $(n=6)$ with specific levels of p-ERK1/2 normalized to the corresponding ERK1/2, TH, and tubulin levels. (B) Representative blot showing the effects of SB-277011-A $(50 \mathrm{nM})$ added to the cultures 20 minutes before challenge with nicotine $(10 \mu \mathrm{M})$ assessed after 2 minutes. Right panel, densitometric analysis of blots $(n=6)$ normalized as described. (C) Representative blot showing the effects of LY294002 (10 $\mu \mathrm{M})$ added to the cultures 20 minutes before challenge with nicotine $(10 \mu \mathrm{M})$ assessed after 2 minutes. Right panel, densitometric analysis of blots $(n=6)$ normalized as described. (D-R) Immunofluorescence microphotographs of TH-IR (D, G, J, M, and P), p-ERK-IR (E, H, K, N, and Q), and their colocalization (F, I, L, O, and R) in mesencephalic dopaminergic neurons 2 minutes after treatment with nicotine (D-F) and SB-277011-A (20 minutes), followed by nicotine (G-I), SB-277011-A alone (J-L), and LY294002, followed by nicotine (MO) or LY294002 alone (P-R). Cell nuclei were stained with Hoechst. Scale bar: $20 \mu \mathrm{m}$. (S) Representative blot showing the effects of vehicle, nicotine, and 7-OH-DPAT on p-ERK1/2 in mesencephalic cultures from wild-type and D3KO mice. Right panel, densitometric analysis of blots $(n=4)$ normalized as described. V, vehicle; N, nicotine; SB, SB-277011-A; Ly, LY294002; 7OH, 7-OH-DPAT. Densitometric values were represented as percentage of vehicle or nicotine values as mean \pm S.E.M. $\left({ }^{* * *} P<0.001 ; * * P<0.01 ; * P<0.05\right.$ versus vehicle; ${ }^{\circ * o} P<0.001 ;{ }^{\circ} P<0.01$ versus nicotine post-hoc Bonferroni test).

plasticity of mesencephalic dopaminergic neurons was tested in independent experiments. Nicotine $(10 \mu \mathrm{M})$ was administered after pretreatments with the pharmacologic inhibitors LY294002 (10 $\mu \mathrm{M})$, rapamycin (2 nM), or PD98059 (10 $\mu \mathrm{M})$, which block phosphorylation of PI3K, mTORC1, and mitogenactivated protein kinase kinase (MEK), respectively. Three days later, maximal dendrite length showed a significant treatment effect, mostly because of a reliable increase produced by nicotine (Bonferroni's test $P<0.001$ ) that was attenuated in various experiments by pretreatment with either LY294002
$[F(3,127)=7.30 ; P<0.0002 ;$ Bonferroni's test $P<0.01]$ (Fig. 8A), rapamycin $[F(3,159)=8.71 ; P<0.0001$; Bonferroni's test $P<0.01]$ (Fig. 8D), or PD98059 $[F(3,123)=9.10 ; P<$ 0.0001; Bonferroni's test $P<0.01$ ] (Fig. 8G).

Similar significant outcomes were observed for the number of primary dendrites; the enhancing effects of nicotine (Bonferroni's test $P<0.01$ ) were blocked by pretreatments with LY294002 $[F(3,122)=6.11 ; P<0.001$; Bonferroni's test $P<0.01]$ (Fig. 8B), rapamycin $[F(3,199)=5.67 ; P<0.001$; Bonferroni's test $P<0.05]$ (Fig. $8 \mathrm{E}$ ), and PD98059 $[F(3,119)=5.66 ; P<0.002$; 
A

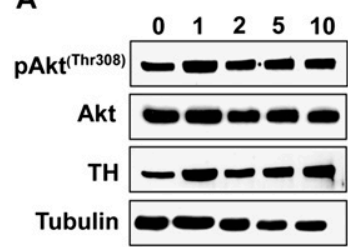

B
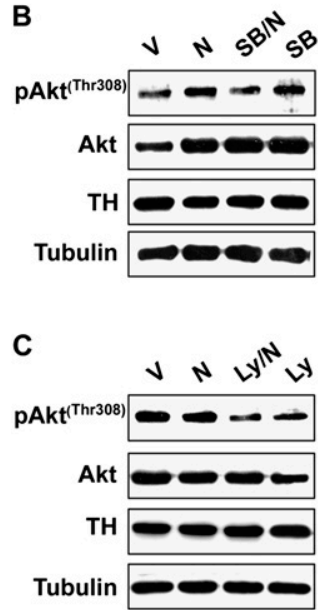

S

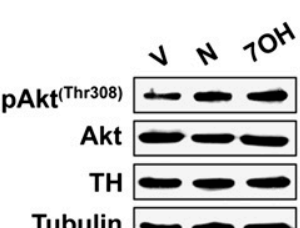

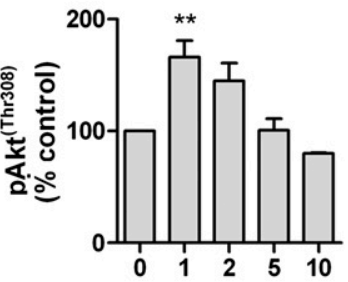
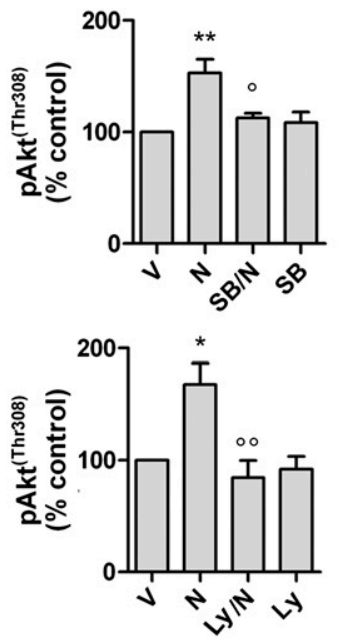

D3KO

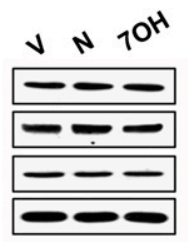

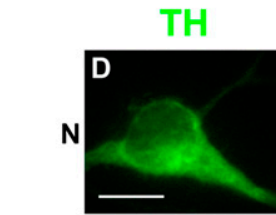
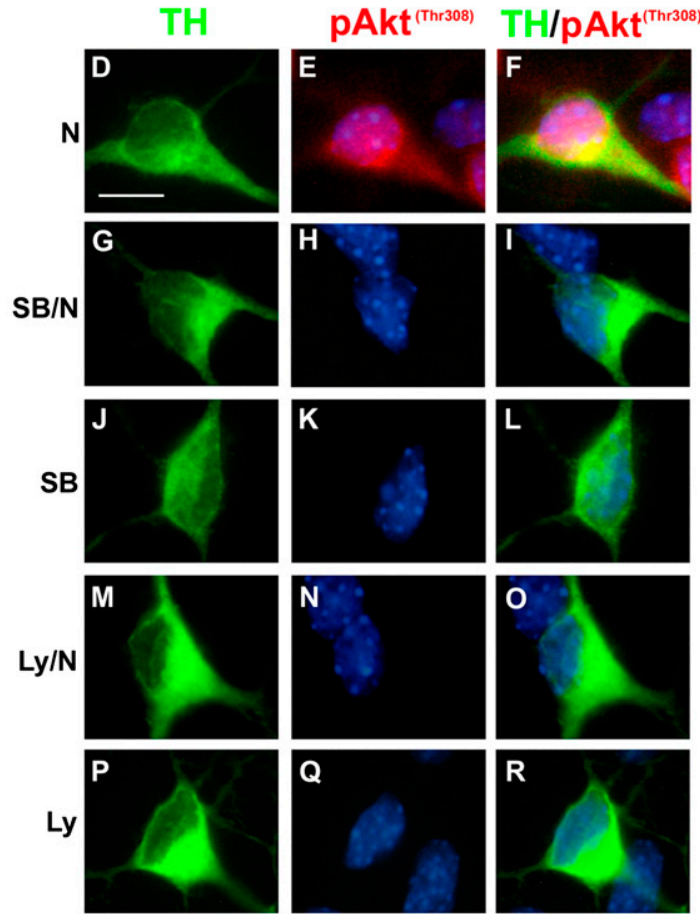

Fig. 6. Akt phosphorylation activated by nicotine challenge in mesencephalic TH-IR neurons is prevented by D3R antagonist SB-277011-A and PI3K inhibitor LY294002 and is blocked in D3KO mice. (A) Representative time course of Akt phosphorylation induced by nicotine (10 $\mu$ M) at $0,1,2,5$, and 10 minutes; cell lysates were processed for Western blot analysis that was performed using antibodies to detect pAkt, Akt, TH, and tubulin levels; membranes were stripped and reprobed as described in Materials and Methods. Right panel, densitometric analysis of blots $(n=6)$ with specific levels of p-Akt normalized to the corresponding Akt, TH, and tubulin levels. (B) Representative blot showing the effects of SB-277011-A (50 nM) added to the cultures 20 minutes before challenge with nicotine $(10 \mu \mathrm{M})$ assessed after 1 minute. Right panel, densitometric analysis of blots $(n=5)$ normalized as described. (C) Representative blot showing the effects of LY294002 (10 $\mu \mathrm{M})$ added to the cultures 20 minutes before challenge with nicotine (10 $\mu \mathrm{M})$ assessed after 1 minute. Right panel, densitometric analysis of blots $(n=5)$ normalized as described. (D-R) Immunofluorescence microphotographs of TH-IR (D, G, J, M, and P), p-Akt-IR (E, H, K, N, and Q), and their colocalization (F, I, L, O, and R) in mesencephalic dopaminergic neurons 1 minute after treatment with nicotine (D-F), SB-277011-A (20 minute) followed by nicotine (G-I), SB-277011-A alone (J-L), LY294002 followed by nicotine (M-O), and LY294002 alone (P-R). Cell nuclei were stained by Hoechst. Scale bar: $20 \mu \mathrm{m}$. (S) Representative blot showing the effects of vehicle, nicotine, and 7-OHDPAT on p-Akt in mesencephalic cultures from wild-type and D3KO mice. Right panel, densitometric analysis of blots $(n=3)$ normalized as described. V, vehicle; N, nicotine; SB, SB-277011-A; Ly, LY294002; 7OH, 7-OH-DPAT. Densitometric values were represented as percentage of vehicle or nicotine values as mean \pm S.E.M. $\left({ }^{* *} P<0.01 ;{ }^{*} P<0.05\right.$ versus vehicle; ${ }^{\circ} P<0.01 ;{ }^{\circ} P<0.05$ versus nicotine post-hoc Bonferroni test).

Bonferroni's test $P<0.05]$ (Fig. $8 \mathrm{H}$ ). The same pattern was observed by measuring the changes in TH-IR soma area, with increments produced by nicotine (Bonferroni's test $P<$ $0.01)$ that were antagonized by pretreatment with LY294002 $[F(3,141)=10.86 ; P<0.0001 ;$ Bonferroni's test $P<0.001]$ (Fig. 8C), rapamycin $[F(3,159)=7.23 ; P<0.0001$; Bonferroni's test $P<0.01]$ (Fig. 8F), and PD98059 $[F(3,137)=6.01 ; P<$ 0.001; Bonferroni's test $P<0.05$ ] (Fig. 8I).

Overall, these data suggest that both the PI3K-Akt-mTORC1 and the PI3K-MEK-ERK signaling pathways are necessary to mediate the structural plasticity effects induced by nicotine in mesencephalic dopaminergic neurons.

\section{Discussion}

In the present study, we showed that prolonged exposure to nicotine produced structural plasticity in mesencephalic DAergic neurons of the mouse embryo by increasing soma area and dendrite arborisation via a mechanism dependent on D3R activation by engaging of ERK and Akt-mTOR signaling pathways. These findings support evidence of nicotinedependent structural plasticity in the DAergic system (Janson et al., 1988a,b; Bunnemann et al., 2000; Mudo et al., 2007) and clarify the role of specific intracellular pathways involved in growth and survival (Doura et al., 2010; Quik et al., 2012). In our work, nicotine produced structural changes in DAergic 
A
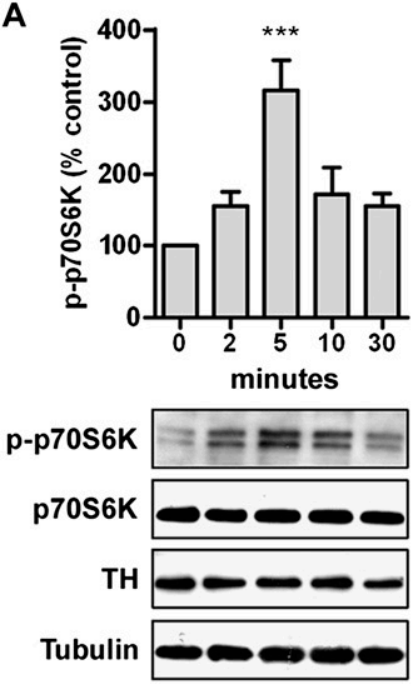

B
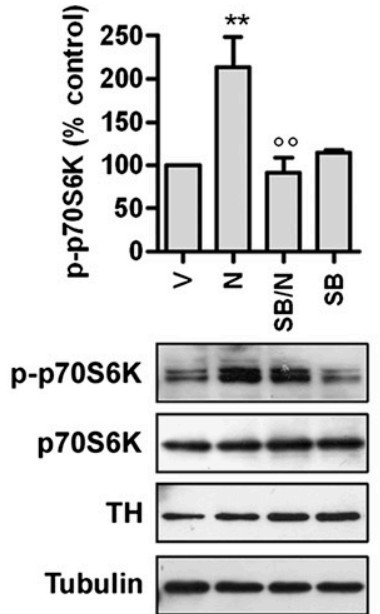

C
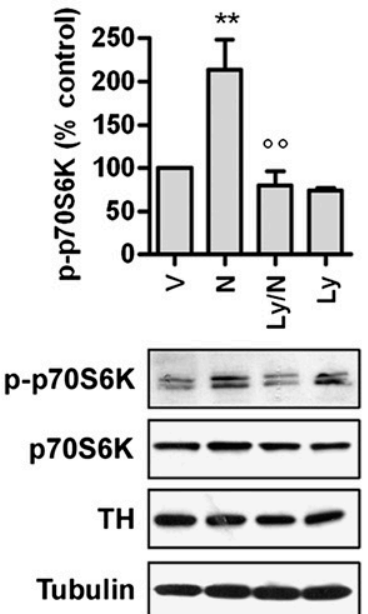
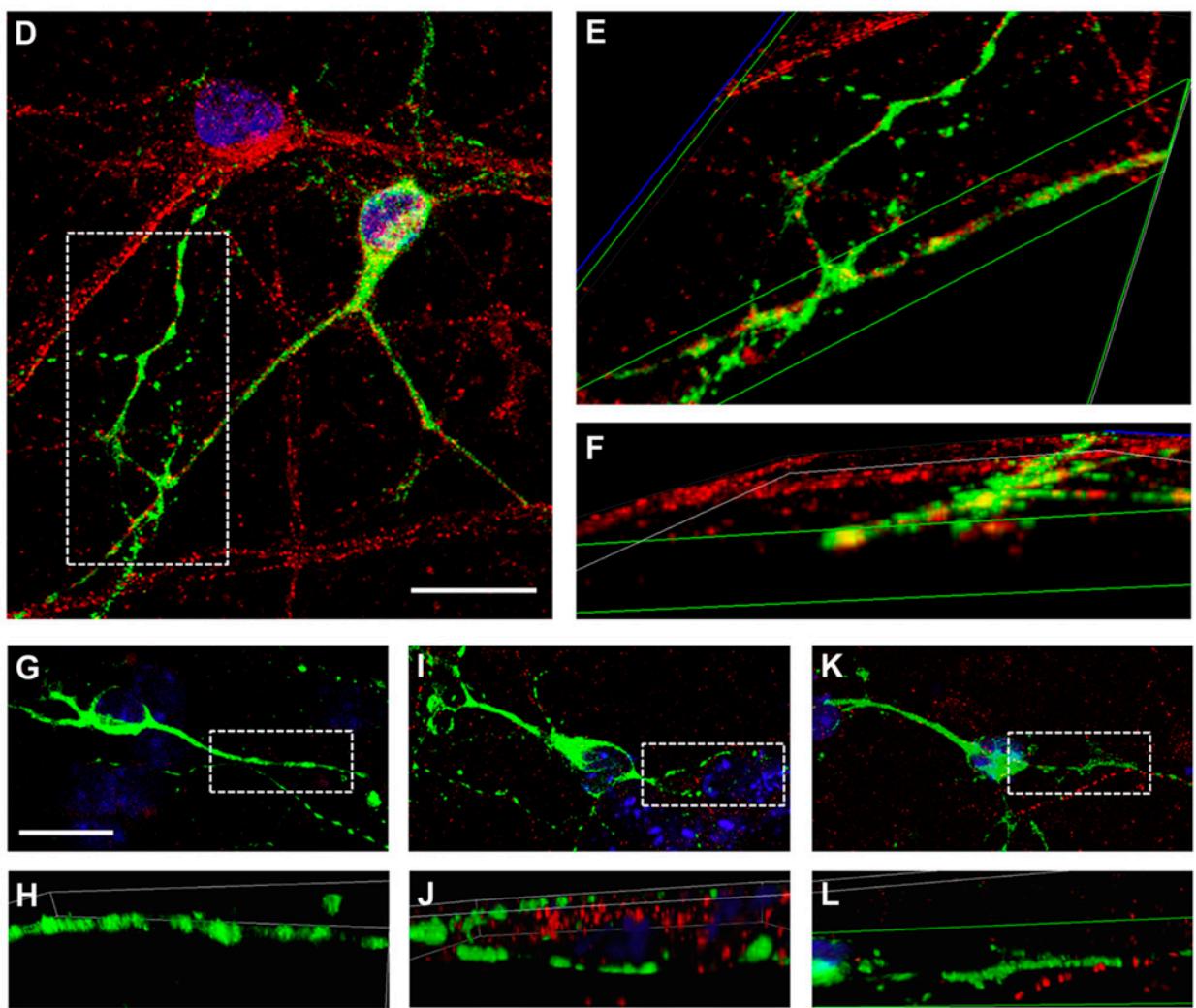
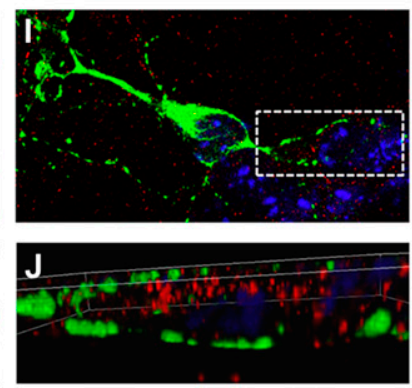
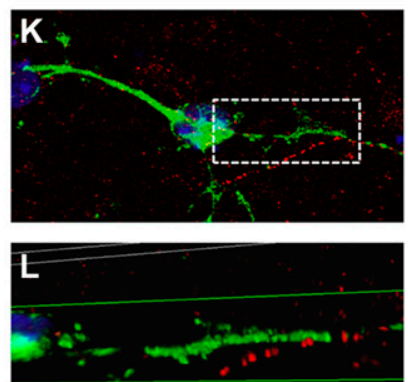

Fig. 7. p70S6K phosphorylation activated by nicotine challenge in mesencephalic $\mathrm{TH}$ IR neurons is prevented by D3R antagonist SB-277011-A and PI3K inhibitor LY294002. (A) Time course of p70S6K phosphorylation induced by nicotine $(10 \mu \mathrm{M})$ at $0,2,5,10$, and 30 minutes; upper panel, densitometric analysis of blots $(n=5)$ with specific levels of p-p70S6K, normalized to the corresponding p70S6K, TH, and tubulin levels; lower panel representative Western blot; cell lysates were processed for Western blot analysis that was performed using antibodies to detect p-p70S6K, p70S6K, TH, and tubulin levels; membranes were stripped and reprobed as described in Materials and Methods. (B) Effect of SB-277011-A (50 $\mathrm{nM}$ ) added to the cultures 20 minutes before challenge with nicotine $(10 \mu \mathrm{M})$ assessed after 5 minutes; upper panel, densitometric analysis of blots $(n=6)$ normalized as described; lower panel, representative Western blot. (C) Effects of LY294002 $(10 \mu \mathrm{M})$ added to the cultures 20 minutes before challenge with nicotine $(10 \mu \mathrm{M})$ assessed after 5 minutes; upper panel, densitometric analysis of blots $(n=6)$ normalized as described; lower panel, representative Western blot. Densitometric values were represented as percentage of vehicle or nicotine values as mean \pm S.E.M. $(* * * P<$ $0.001 ; * * P<0.01$ versus vehicle; ${ }^{\circ} P<$ 0.01 versus nicotine post-hoc Bonferroni test); $\mathrm{V}$, vehicle; N, nicotine; SB, SB-277011-A; Ly, LY294002. (D-F) Confocal analysis of THIR neurons showing that $\mathrm{p}$-p70S6K appears in the soma and in distinct punctae along dendrites 5 minutes after nicotine $(10 \mu \mathrm{M})$ treatment. p-p70S6K (rabbit polyclonal antip-p70S6K, Cy3-red), TH (mouse monoclonal anti-TH, Alexa Fluor 488-green). A high magnification of a representative dendrite is shown in its Z-stack projection on xy plane (E) and on yz plane (F). (G-H) TH-IR neurons treated with vehicle $(\mathrm{G})$ and a Z-stack projection of a dendrite on xy plane (H); (I-J) pre-treatment of TH-IR neurons with SB-277011-A followed by nicotine $(10 \mu \mathrm{M})(\mathrm{I})$ and a Z-stack projection of a dendrite on xy plane (J). (K-L) Pretreatment of TH-IR neurons with LY294002 followed by nicotine $(10 \mu \mathrm{M})(\mathrm{K})$ and a Z-stack projection of a dendrite on xy plane (L). Scale bar: $20 \mu \mathrm{m}$ neurons either when added to primary cultures obtained from the mesencephalon of E12.5 mouse embryos or when administered to pregnant mice at about the same stage of embryo development and studied in the VTA and SN of P1 offspring. In vitro studies in primary neurons were conducted using a validated protocol that generates almost pure neuronal cultures containing 5-7\% dopaminergic neurons and less than 0.5\% astrocytes (Collo et al., 2008). Nicotine produced a dosedependent effect on maximal dendrite length and on the number of primary dendrites starting at the dose of $1 \mu \mathrm{M}$, levels observed in smokers and compatible with in vivo pharmacologic effects in mice (Changeux, 2010). In the present work, we chose to use the dose of $10 \mu \mathrm{M}$ to maximize the pharmacological signal of the test. This effect was detectable at 12-24 hours and stabilized to the highest value at 72 hours of incubation. The delayed effect on neuronal soma was also observed after cocaine exposure (Collo et al., 2012), a phenomenon probably related to the soma geometry and/or to specific molecular substrates engaged in growth (Scheidenhelm et al., 2005; Russo et al., 2010). The involvement of $\alpha 4 \beta 2 * \mathrm{nAChR}$ in mediating nicotine's effects was supported by the blockade of dendrite and soma growth produced by the nonselective antagonist MEC and by the $\beta 2 *$-selective antagonist $\mathrm{DH} \beta \mathrm{E}$, whereas no effects were observed with the $\alpha 7 \mathrm{nAChR}$ antagonist MLA. This argument is further supported by the lack of response to nicotine of primary cultures from embryos of 
A

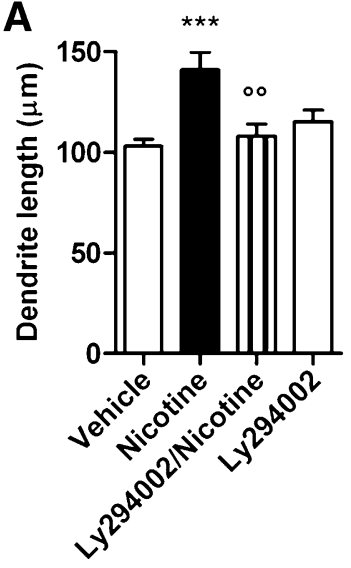

D
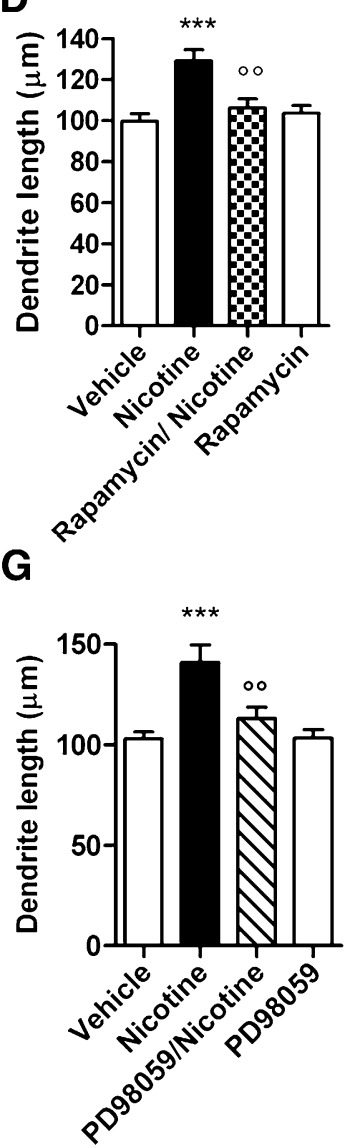

B

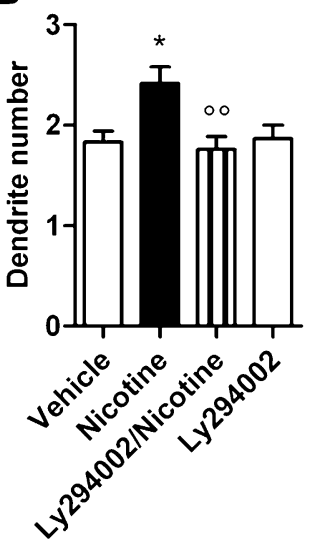

E
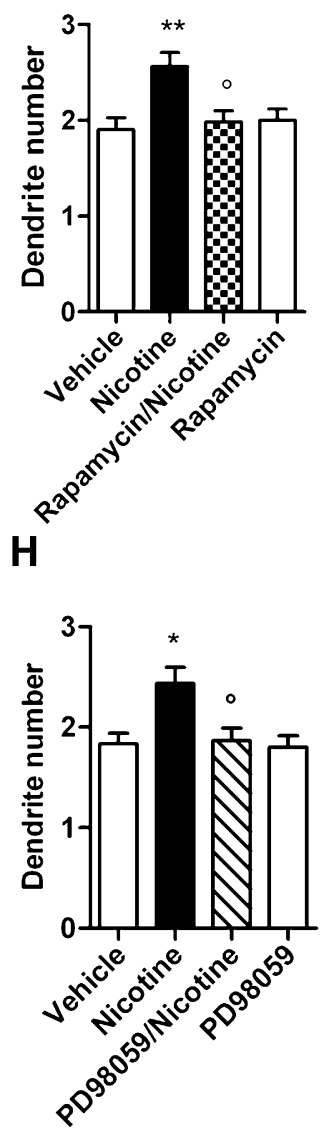

C

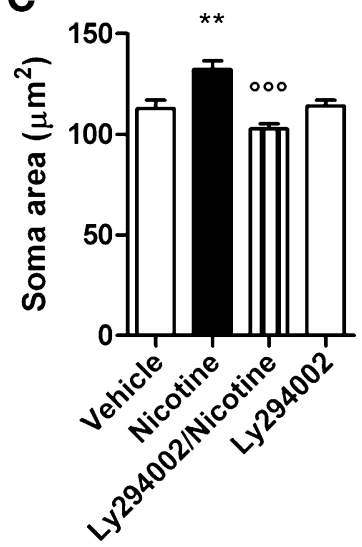

$\mathbf{F}$
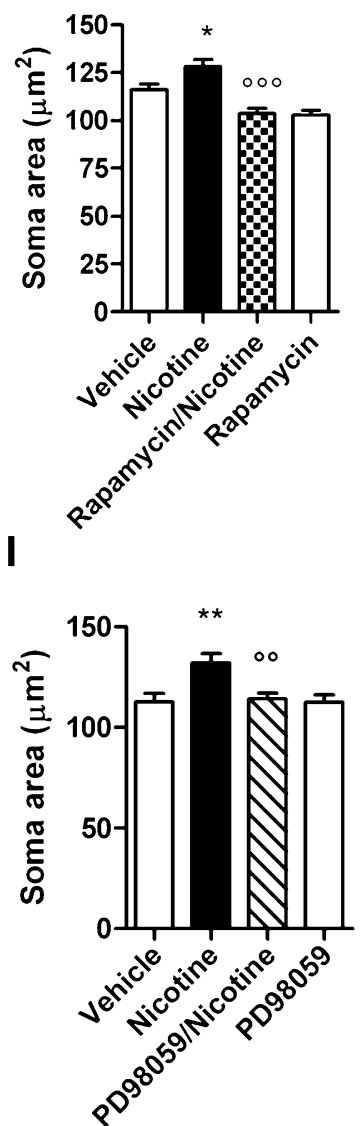

Fig. 8. Nicotine-induced structural plasticity in dopaminergic neurons is prevented by PI3K inhibitor LY294002, mTORC1 inhibitor rapamycin, and MEK inhibitor PD98059. (A-C) Morphologic effects of pretreatment (20 minutes) with LY294002 $(10 \mu \mathrm{M})$ on structural plasticity produced by nicotine $(10 \mu \mathrm{M})$ assessed as (A) maximal dendrite length, (B) number of primary dendrites, and (C) soma area. (D-F) Morphologic effects of pretreatment (10 minutes) with rapamycin $(10 \mathrm{nM})$ followed by three washes and incubation with nicotine $(10 \mu \mathrm{M})$ dissolved in fresh medium: (D) maximal dendrite length, (E) number of primary dendrites, and (F) soma area. (G-I) Morphologic effects of pretreatment (20 minutes) with PD98059 $(10 \mu \mathrm{M})$ followed by nicotine $(10 \mu \mathrm{M})$ : $(\mathrm{G})$ maximal dendrite length, $(\mathrm{H})$ number of primary dendrites, and (I) soma area. All measurements were performed after 72 hours. $(* * * P<0.001 ; * * P<0.01 ; * P<0.05$ versus vehicle; ${ }^{\circ o o} P<0.001 ;{ }^{\circ 0} P<0.01 ;{ }^{\circ} P<0.05$ versus nicotine; post-hoc Bonferroni test). $\alpha 4 \mathrm{KO}$ mice, whereas significant response was seen with BDNF, suggesting the presence of a normal signaling to neurotrophic stimuli. The lack of response to nicotine was interpreted as attributable to the absence of heteromeric $\alpha 4^{*} \mathrm{nAChR}$, the most commonly expressed $\mathrm{nAChR}$ in mesencephalic dopaminergic neurons (Champtiaux et al., 2003; Marubio et al., 2003) since early embryonic stages (Zoli et al., 1995). Converging findings indicate that the $\alpha 4 \beta 2^{*} \mathrm{nAChR}$ participates to the control of DAergic neuron firing in the VTA and of DA release from DAergic terminals in ventral striatum (Picciotto et al., 1998; Wonnacott et al., 2000). These $\alpha 4 \beta 2^{*} \mathrm{nAChR}$ regulatory effects on the mesencephalic DA system are critically involved in mediating the reinforcing properties of nicotine (Picciotto et al., 1998; Marubio et al., 2003; Changeux, 2010), evidence supported by the effects of the $\alpha 4 \beta 2 *$ nAChR partial agonist varenicline in humans and mice (Rollema et al., 2009; Reperant et al., 2010). A role of $\alpha 4 \beta 2^{*} \mathrm{nAChR}$ in PD was recently suggested (Mudo et al., 2007; Quik et al., 2012). For example, varenicline reduced L-3,4-dihydroxyphenylalanine (L-DOPA)induced abnormal involuntary movements in rats with partial striatal dopamine lesion in a model of PD (Huang et al., 2011). However, because of the higher response obtained with the $10 \mu \mathrm{M}$ nicotine dose, with respect to the $1 \mu \mathrm{M}$ dose, we cannot exclude the involvement of receptors other than high affinity non- $\alpha 7$ receptors (Jensen et al., 2005).

The morphologic changes produced by nicotine in primary cultures of mesencephalic neurons resembled those of amphetamine and cocaine (Collo et al., 2008, 2012). In these 
studies, we proposed that the high extracellular DA levels produced by amphetamine and cocaine were involved in structural plasticity by activating D3 autoreceptors that are expressed in all TH-positive neurons of the mesencephalon (Diaz et al., 2000). Indeed, we showed that psychostimulant effects were blocked by a low dose of D3R antagonist SB-277011-A and did not occur in cultures from D3KO mice. Structural plasticity was produced by D3Rpreferential agonists, such as 7-OH-DPAT, quinpirole, and ropinerole, all anti-Parkinsonian agents capable of driving regeneration of mesencephalic DAergic neurons after damage in vivo (Van Kampen and Eckman, 2006; Joyce and Millan, 2007).

In the present article, we propose that the activation of the D3 autoreceptors was also critical for generating nicotinedependent structural plasticity and that these effects were possibly mediated by DA released by nicotine. Accordingly, nicotine-elicited somatodendritic release of DA in VTA was described in vitro and in vivo (Rahman et al., 2004; Kleijn et al., 2011), and preliminary findings using highperformance liquid chromatography measurements indicated that DA levels were increased by nicotine in the culture media of mesencephalic neurons (Collo, unpublished data). This hypothesis is indirectly supported by the evidence that nicotine-induced structural plasticity was completely prevented by the D3R-preferential antagonist SB-277011-A and was absent in mesencephalic cultures prepared from D3KO embryos, whereas BDNF was still effective. Of note, this interpretation is compatible with the well-established role of BDNF in controlling D3 receptor expression (Le Foll et al., 2005) and suggests a reciprocal positive-feedback relationship in generating neurotrophic effects. Receptor-receptor interaction between D3R and $\alpha 4 \beta 2^{*}$ nAChR could also occur, as suggested by the recently described D3R synergistic interaction with D1R (Fiorentini et al., 2008) and the proposed dimerization between non- $\alpha 7^{*} \mathrm{nAChR}$ and D2 autoreceptors in striatal dopaminergic terminals (Quarta et al., 2007). However, it is possible that D3R IR expressed in other neurons of SN and VTA that do not contain DA could mediate some of these effects by engaging other neurotransmitters (e.g., glutamate) (Sokoloff et al., 2013).

Similar effects were also observed in vivo by exposing mouse embryos to nicotine during the late phase of pregnancy. We observed that nicotine increased the soma size of DAergic neurons of both VTA and SN in newborn wild-type mice. The same treatment did not produce any effect in the D3KO offspring. These results were in line with those obtained after prenatal exposure to cocaine (Collo et al., 2012), suggesting that some commonalities between nicotine and cocaine in generating structural plasticity of DAergic neurons can be related to the activation of D3R. Incidentally, prenatal exposure to nicotine and cocaine in human and rodents was associated with long-lasting postnatal neurobiological and behavioral effects (Crozatier et al., 2003; Bublitz and Stroud, 2012).

The intracellular pathways that drive the effects of nicotine on dendritic growth and soma expansion in DAergic neurons were investigated in primary mesencephalic cell cultures with use of Western blot and dual-staining immunofluorescence to localize phosphorylation events in TH-IR-positive profiles. Exposure to nicotine induced phosphorylation of ERK and of Akt within 1-2 minutes, suggesting a rapid triggering of two of the main signaling pathways involved in dendritic growth and structural plasticity (Alonso et al., 2004; Jaworski et al., 2005; Kumar et al., 2005; Scheidenhelm et al., 2005). Both MEK-ERK and PI3K-Akt-mTOR pathways are known to be activated by BDNF via Ras acting as common activator (McAllister et al., 1999). Of interest, transactivation between IGF1 and D3R was described in transfected CHO cells, suggesting a possible highjacking of neurotrophin intracellular signaling (Mannoury la Cour et al., 2011). Previously, using heterologous transfection of D3R, Cussac et al. (1999) and, later, Beom et al. (2004) showed that D3Rs phosphorylate both ERK and PI3K-Akt pathways by engaging PI3K. This mechanism was recently found in DAergic neurons with use of direct and indirect DAergic agonists (Collo et al., 2008, 2012). In the present work, we showed that pretreatments with either the PI3K inhibitor LY294002 or the D3R antagonist SB-277011-A completely blocked ERK and Akt phosphorylation induced by nicotine. The effects of nicotine were not observed in mesencephalic neurons obtained from D3KO mice, further supporting a key role of D3R activation as mediator of nicotine effect. Furthermore, for the first time to our knowledge, we provide evidence that in DAergic neurons the activation of both pathways is required to trigger the phosphorylation of p70S6K, preferred substrate of mTORC1 involved in ribosomal biogenesis and protein translation (Jefferies et al., 1997; Kumar et al., 2005). The peak time of p70S6K phosphorylation followed that of ERK and Akt, supporting its downstream position in the signaling cascade. Confocal microscopy showed that nicotine-induced p-p70S6K was present in the soma and in micro-domains of the dendrites of DA neurons that do not contain TH-IR. Phosphorylation of p70S6K was prevented by pretreatments with the selective inhibitor of $\mathrm{mTORC} 1$ rapamycin (Jefferies et al., 1997; Jaworski et al., 2005). These findings are in line with the early observation of dendritic micro-domain activation of p70S6K induced by $N$-methyl-D-aspartate in hippocampal neurons (Cammalleri et al., 2003). The present data strongly support the hypothesis that the observed nicotineelicited activation of MEK-ERK and PI3K-Akt-mTOR pathways mediates structural plasticity, because rapamycin, the PI3K inhibitor LY294002, or the MEK inhibitor PD98059 blocked nicotine-induced dendrite growth and soma size increase.

In conclusion, although the relevance of the present observations to the influence of nicotine on dopaminergic pathways and cellular signals in adult neurons remains to be confirmed, they suggest a crucial role for D3R and Akt-mTOR signaling in the expression of nicotine-induced structural plasticity in mesencephalic dopaminergic neurons. As such, they provide a template for further analysis of the role of nicotinic and dopaminergic mechanisms in addiction on the one hand (mesolimbic dopaminergic neurons) and in neuroprotection and motor behavior on the other (nigrostriatal pathways). Because recent positron emission tomography findings indicate that D3R are particularly enriched in human SN and VTA (Searle et al., 2010), our findings may suggest novel strategies for the treatment of both addiction and PD.

\section{Acknowledgments}

The authors thank Marzia Melotti for technical support and Cecilia Gotti for the gifts of mecamylamine, dihydro- $\beta$-erythroidine, and methyllycaconitine. 


\section{Authorship Contributions}

Participated in research design: Collo, Merlo Pich, Zoli, Missale. Conducted experiments: Collo, Bono, Cavalleri, Plebani, Mitola. Contributed new reagents or analytic tools: Millan, Maskos.

Performed data analysis: Collo, Bono, Cavalleri, Plebani, Mitola, Merlo Pich.

Wrote or contributed to the writing of the manuscript: Collo, Merlo Pich, Millan, Zoli, Maskos, Spano, Missale.

\section{References}

Accili D, Fishburn CS, Drago J, Steiner H, Lachowicz JE, Park B-H, Gauda EB, Lee EJ, Cool MH, and Sibley DR et al. (1996) A targeted mutation of the D3 dopamine receptor gene is associated with hyperactivity in mice. Proc Natl Acad Sci USA 93 1945-1949.

Alonso M, Medina JH, and Pozzo-Miller L (2004) ERK1/2 activation is necessary for $\mathrm{BDNF}$ to increase dendritic spine density in hippocampal CA1 pyramidal neurons. Learn Mem 11:172-178.

Benowitz NL (1988) Drug therapy. Pharmacologic aspects of cigarette smoking and nicotine addition. $N$ Engl J Med 319:1318-1330.

Beom SR, Cheong D, Torres G, Caron MG, and Kim KM (2004) Comparative studies of molecular mechanisms of dopamine D2 and D3 receptors for the activation of extracellular signal-regulated kinase. J Biol Chem 279:28304-28314.

Bublitz MH and Stroud LR (2012) Maternal smoking during pregnancy and offspring brain structure and function: review and agenda for future research. Nicotine Tob Res 14:388-397.

Bunnemann B, Terron A, Zantedeschi V, Merlo Pich E, and Chiamulera C (2000) Chronic nicotine treatment decreases neurofilament immunoreactivity in the rat ventral tegmental area. Eur J Pharmacol 393:249-253.

Cammalleri M, Lütjens R, Berton F, King AR, Simpson C, Francesconi W, and Sanna PP (2003) Time-restricted role for dendritic activation of the mTOR-p70S6K pathway in the induction of late-phase long-term potentiation in the CA1. Proc Natl Acad Sci USA 100:14368-14373.

Champtiaux N, Gotti C, Cordero-Erausquin M, David DJ, Przybylski C, Léna C, Clementi F, Moretti M, Rossi FM, and Le Novère N et al. (2003) Subunit composition of functional nicotinic receptors in dopaminergic neurons investigated with knock-out mice. J Neurosci 23:7820-7829.

Changeux JP (2010) Nicotine addiction and nicotinic receptors: lessons from genetically modified mice. Nat Rev Neurosci 11:389-401.

Collo G, Zanetti S, Missale C, and Spano PF (2008) Dopamine D3 receptor-preferring agonists increase dendrite arborization of mesencephalic dopaminergic neurons via extracellular signal-regulated kinase phosphorylation. Eur $J$ Neurosci 28 $1231-1240$

Collo G, Bono F, Cavalleri L, Plebani L, Merlo Pich E, Millan MJ, Spano PF, and Missale C (2012) Pre-synaptic dopamine D(3) receptor mediates cocaineinduced structural plasticity in mesencephalic dopaminergic neurons via ERK and Akt pathways. $J$ Neurochem 120:765-778.

Corrigall WA, Coen KM, and Adamson KL (1994) Self-administered nicotine activates the mesolimbic dopamine system through the ventral tegmental area. Brain Res 653:278-284.

Crozatier C, Guerriero RM, Mathieu F, Giros B, Nosten-Bertrand M, and Kosofsky $\mathrm{BE}$ (2003) Altered cocaine-induced behavioral sensitization in adult mice exposed to cocaine in utero. Brain Res Dev Brain Res 147:97-105.

Cussac D, Newman-Tancredi A, Pasteau V, and Millan MJ (1999) Human dopamine $\mathrm{D}(3)$ receptors mediate mitogen-activated protein kinase activation via a phosphatidylinositol 3-kinase and an atypical protein kinase C-dependent mechanism. Mol Pharmacol 56:1025-1030.

Diaz J, Pilon C, Le Foll B, Gros C, Triller A, Schwartz J-C, and Sokoloff P (2000) Dopamine D3 receptors expressed by all mesencephalic dopamine neurons. $J$ Neurosci 20:8677-8684.

Doura MB, Luu TV, Lee NH, and Perry DC (2010) Persistent gene expression changes in ventral tegmental area of adolescent but not adult rats in response to chronic nicotine. Neuroscience 170:503-513.

Fiorentini C, Busi C, Gorruso E, Gotti C, Spano PF, and Missale C (2008) Reciprocal regulation of dopamine D1 and D3 receptor function and trafficking by heterodimerization. Mol Pharmacol 74:59-69.

Grandinetti A, Morens DM, Reed D, and MacEachern D (1994) Prospective study of cigarette smoking and the risk of developing idiopathic Parkinson's disease. Am J Epidemiol 139:1129-1138.

Heidbreder CA, Gardner EL, Xi Z-X, Thanos PK, Mugnaini M, Hagan JJ, and Ashby $\mathrm{CR}, \mathrm{Jr}(2005)$ The role of central dopamine D3 receptors in drug addiction: a review of pharmacological evidence. Brain Res Brain Res Rev 49:77-105.

Huang LZ, Campos C, Ly J, Ivy Carroll F, and Quik M (2011) Nicotinic receptor agonists decrease L-dopa-induced dyskinesias most effectively in partially lesioned parkinsonian rats. Neuropharmacology 60:861-868

Hyman C, Hofer M, Barde YA, Juhasz M, Yancopoulos GD, Squinto SP, and Lindsay RM (1991) BDNF is a neurotrophic factor for dopaminergic neurons of the substantia nigra. Nature 350:230-232.

Kleijn J, Folgering JH, van der Hart MC, Rollema H, Cremers TI, and Westerink BH (2011) Direct effect of nicotine on mesolimbic dopamine release in rat nucleus accumbens shell. Neurosci Lett 493:55-58.

Kumar V, Zhang M-X, Swank MW, Kunz J, and Wu G-Y (2005) Regulation of dendritic morphogenesis by Ras-PI3K-Akt-mTOR and Ras-MAPK signaling pathways. J Neurosci 25:11288-11299.

Kwon CH, Zhu X, Zhang J, and Baker SJ (2003) mTor is required for hypertrophy of Pten-deficient neuronal soma in vivo. Proc Natl Acad Sci USA 100:12923-12928. Janson AM, Fuxe K, Sundström E, Agnati LF, and Goldstein M (1988a) Chronic nicotine treatment partly protects against the 1-methyl-4-phenyl-2,3,6-tetrahydropyridine- induced degeneration of nigrostriatal dopamine neurons in the black mouse. Acta Physiol Scand 132:589-591.

Janson AM, Fuxe K, Agnati LF, Kitayama I, Härfstrand A, Andersson K, and Goldstein M (1988b) Chronic nicotine treatment counteracts the disappearance of tyrosine-hydroxylase-immunoreactive nerve cell bodies, dendrites and terminals in the mesostriatal dopamine system of the male rat after partial hemitransection. Brain Res 455:332-345.

Jaworski J, Spangler S, Seeburg DP, Hoogenraad CC, and Sheng M (2005) Control of dendritic arborization by the phosphoinositide-3'-kinase-Akt-mammalian target of rapamycin pathway. J Neurosci 25:11300-11312.

Jefferies HB, Fumagalli S, Dennis PB, Reinhard C, Pearson RB, and Thomas G (1997) Rapamycin suppresses 5'TOP mRNA translation through inhibition of p70s6k. EMBO J 16:3693-3704.

Jensen AA, Frølund B, Liljefors T, and Krogsgaard-Larsen P (2005) Neuronal nicotinic acetylcholine receptors: structural revelations, target identifications, and therapeutic inspirations. J Med Chem 48:4705-4745.

Joyce JN and Millan MJ (2007) Dopamine D3 receptor agonists for protection and repair in Parkinson's disease. Curr Opin Pharmacol 7:100-105.

Le Foll B, Diaz J, and Sokoloff P (2005) A single cocaine exposure increases BDNF and D3 receptor expression: implications for drug-conditioning. Neuroreport 16:175-178.

Le Foll B, Goldberg SR, and Sokoloff P (2007) Dopamine D3 receptor ligands for the treatment of tobacco dependence. Expert Opin Investig Drugs 16:45-57.

Mannoury la Cour C, Salles MJ, Pasteau V, and Millan MJ (2011) Signaling pathways leading to phosphorylation of Akt and GSK-3 $\beta$ by activation of cloned human and rat cerebral $\mathrm{D}_{2}$ and $\mathrm{D}_{3}$ receptors. Mol Pharmacol 79:91-105.

Marubio LM, Gardier AM, Durier S, David D, Klink R, Arroyo-Jimenez MM, McIntosh JM, Rossi F, Champtiaux N, and Zoli M et al. (2003) Effects of nicotine in the dopaminergic system of mice lacking the alpha4 subunit of neuronal nicotinic acetylcholine receptors. Eur J Neurosci 17:1329-1337.

McAllister AK, Katz LC, and Lo DC (1999) Neurotrophins and synaptic plasticity. Annu Rev Neurosci 22:295-318.

Missale C, Nash SR, Robinson SW, Jaber M, and Caron MG (1998) Dopamine receptors: from structure to function. Physiol Rev 78:189-225.

Mudo G, Belluardo N, and Fuxe K (2007) Nicotinic receptor agonists as neuroprotective/ neurotrophic drugs. Progress in molecular mechanisms. J Neural Transm 114:135-147.

Mueller D, Chapman CA, and Stewart J (2006) Amphetamine induces dendritic growth in ventral tegmental area dopaminergic neurons in vivo via basic fibroblast growth factor. Neuroscience 137:727-735.

Nestler EJ, Hope BT, and Widnell KL (1993) Drug addiction: a model for the molecular basis of neural plasticity. Neuron 11:995-1006.

Picciotto MR, Zoli M, Rimondini R, Léna C, Marubio LM, Pich EM, Fuxe K, and Changeux JP (1998) Acetylcholine receptors containing the beta2 subunit are involved in the reinforcing properties of nicotine. Nature 391:173-177.

Quarta D, Ciruela F, Patkar K, Borycz J, Solinas M, Lluis C, Franco R, Wise RA, Goldberg SR, and Hope BT et al. (2007) Heteromeric nicotinic acetylcolinedopamine autoreceptor complexes modulate striatal dopamine release. Neuropsycopharmacology 32:35-42.

Quik M, Parameswaran N, McCallum SE, Bordia T, Bao S, McCormack A, Kim A, Tyndale RF, Langston JW, and Di Monte DA (2006) Chronic oral nicotine treatment protects against striatal degeneration in MPTP-treated primates. J Neurochem 98:1866-1875.

Quik M, Perez XA, and Bordia T (2012) Nicotine as a potential neuroprotective agent for Parkinson's disease. Mov Disord 27:947-957.

Rahman S, Zhang J, and Corrigall WA (2004) Local perfusion of nicotine differentially modulates somatodendritic dopamine release in the rat ventral tegmental area after nicotine preexposure. Neurochem Res 29:1687-1693.

Reperant C, Pons S, Dufour E, Rollema H, Gardier AM, and Maskos U (2010) Effect of the $\alpha 4 \beta 2 *$ nicotinic acetylcholine receptor $(\mathrm{nAChR})$ partial agonist varenicline on dopamine release: a study in $\beta 2-/-$ mice with selective re-expression of the $\beta 2$ nAChR subunit in the ventral tegmental area. Neuropharmacology 58:346-350.

Rollema H, Hajós M, Seymour PA, Kozak R, Majchrzak MJ, Guanowsky V, Horner WE, Chapin DS, Hoffmann WE, and Johnson DE et al (2009) Preclinical pharmacology of the alpha4beta $2 \mathrm{nAChR}$ partial agonist varenicline related to effects on reward, mood and cognition. Biochem Pharmacol 78:813-824.

Russo SJ, Dietz DM, Dumitriu D, Morrison JH, Malenka RC, and Nestler EJ (2010) The addicted synapse: mechanisms of synaptic and structural plasticity in nucleus accumbens. Trends Neurosci 33:267-276.

Sarti F, Borgland SL, Kharazia VN, and Bonci A (2007) Acute cocaine exposure alters spine density and long-term potentiation in the ventral tegmental area. Eur $J$ Neurosci 26:749-756.

Scheidenhelm DK, Cresswell J, Haipek CA, Fleming TP, Mercer RW, and Gutmann DH (2005) Akt-dependent cell size regulation by the adhesion molecule on glia occurs independently of phosphatidylinositol 3-kinase and Rheb signaling. Mol Cell Biol 25:3151-3162.

Schmidt U, Beyer C, Oestreicher AB, Reisert I, Schilling K, and Pilgrim C (1996) Activation of dopaminergic D1 receptors promotes morphogenesis of developing striatal neurons. Neuroscience 74:453-460.

Searle G, Beaver JD, Comley RA, Bani M, Tziortzi A, Slifstein M, Mugnaini M, Griffante C, Wilson AA, and Merlo-Pich E et al. (2010) Imaging dopamine D3 receptors in the human brain with positron emission tomography, [11C]PHNO, and a selective D3 receptor antagonist. Biol Psychiatry 68:392-399.

Sokoloff P, Leriche L, Diaz J, Louvel J, and Pumain R (2013) Direct and indirect interactions of the dopamine $\mathrm{D}_{3}$ receptor with glutamate pathways: implications for the treatment of schizophrenia. Naunyn Schmiedebergs Arch Pharmacol 386:107-124.

Van Kampen JM and Eckman CB (2006) Dopamine D3 receptor agonist delivery to a model of Parkinson's disease restores the nigrostriatal pathway and improves locomotor behavior. J Neurosci 26:7272-7280.

Vezina P, McGehee DS, and Green WN (2007) Exposure to nicotine and sensitization of nicotine-induced behaviors. Prog Neuropsychopharmacol Biol Psychiatry 31: 1625-1638. 
Wirdefeldt K, Adami HO, Cole P, Trichopoulos D, and Mandel J (2011) Epidemiology and etiology of Parkinson's disease: a review of the evidence. Eur J Epidemiol 26 (S1): S1-58.

Wonnacott S, Kaiser S, Mogg A, Soliakov L, and Jones IW (2000) Presynaptic nicotinic receptors modulating dopamine release in the rat striatum. Eur J Pharmacol 393:51-58.

Zhao-Shea R, Liu L, Soll LG, Improgo MR, Meyers EE, McIntosh JM, Grady SR, Marks MJ, Gardner PD, and Tapper AR (2011) Nicotine-mediated activation of dopaminergic neurons in distinct regions of the ventral tegmental area. Neuropsychopharmacology 36:1021-1032.
Zoli M, Le Novère N, Hill JA, Jr, and Changeux JP (1995) Developmental regulation of nicotinic ACh receptor subunit mRNAs in the rat central and peripheral nervous systems. J Neurosci 15:1912-1939.

Address correspondence to: Ginetta Collo, Department of Molecular and Translational Medicine, and Woman Health Center-Camillo Golgi Foundation, University of Brescia, Viale Europa 11, 25123 Brescia, Italy. E-mail: collo@ med.unibs.it 